\title{
Traitement moral de la question sociale dans la " société harmonieuse » de Hu Jintao
}

Thomas BOUTONNET

\section{(2) OpenEdition}

Journals

Édition électronique

URL : http://journals.openedition.org/transtexts/271

DOI : 10.4000/transtexts. 271

ISSN : 2105-2549

Éditeur

Gregory B. Lee

Édition imprimée

Date de publication : 1 juin 2009

ISSN : 1771-2084

Référence électronique

Thomas BOUTONNET, « Traitement moral de la question sociale dans la « société harmonieuse » de Hu Jintao », Transtext(e)s Transcultures 跨文本跨文化 [En ligne], 5 | 2009, mis en ligne le 01 juin 2009, consulté le 19 avril 2019. URL : http://journals.openedition.org/transtexts/271 ; DOI : 10.4000/ transtexts. 271 


\section{Transtext(e)s}

\section{Transcultures 跨文本跨}

文化

Journal of Global Cultural Studies

5 | 2009:

Varia

(Re)Inventing "Realities" in China

\section{Traitement moral de la question sociale dans la " société harmonieuse » de $\mathrm{Hu}$ Jintao}

THOMAS BOUTONNET

\section{Résumé}

Chinese society in the beginning of this twenty-first century is undermined by growing socioeconomic and regional inequalities, as China has been disrupted by the reforms launched by Deng Xiaoping since 1978, by the transition from a planned economy to a market economy and by the expansion of a mass consumer society. The discourse of 《harmonious society» (hexie shehui 和谐社会), an official program introduced by President $\mathrm{Hu}$ Jintao in 2005 , took place in this particular context to pacify social unrests. This dissertation aims to dismantle the strategies used by the « harmonious society » and to reveal that this discourse advocates social harmony not by resolving social inequities but by accepting them. Hexie shehui is part of a larger and earlier civilizing process of Chinese underprivileged population, since it appears to be a tool to control and discipline the poor and offers ethics response to social issues.

\section{Texte intégral}

La racine de tous les paralogismes, (...) réside dans la capacité du langage de dire le rien, le néant, de faire exister dans les mots et par les mots ce qui n'existe pas dans les choses (...). Cette capacité potentielle, (...) les agents sociaux (et tout 
spécialement les professionnels de la politique, (...)) ne cessent de l'exploiter, pour le meilleur et pour le pire. ${ }^{1}$

C'est dans un contexte social très sensible, marqué par une forte polarisation de la société entre classes de consommateurs favorisés, aux revenus moyens et supérieurs, et classes défavorisées pauvres, que Hu Jintao 胡锦涛 prend la succession de Jiang Zemin 江泽民 en 2002 à la tête du Parti communiste chinois (PCC). ${ }^{2}$ Après avoir introduit en Chine l'économie de marché (dans un processus maîtrisé et accompagné par le PCC, et favorisant les collusions d'intérêt entre forces économiques et politiques) et la société de consommation (en permettant notamment aux consommateurs urbains d'accéder au "bonheur marchand" en échange d'un conservatisme de "classe" favorable à l'ordre établi), le Parti doit faire face aux dommages collatéraux, tant sociaux qu'environnementaux, que plus de vingt années de "réformes et d'ouverture " (gaige kaifang 改革开放) initiées par Deng Xiaoping 邓小平 ont entraînés. ${ }^{3}$ Si le Parti communiste chinois a réussi, entre 1978 et 2002, à basculer la Chine dans une économie de marché, à restructurer l'ensemble du processus de production, auparavant régulé par une économie planifiée, et à " plier » la population chinoise aux règles du capitalisme et du productivisme, « la "mise au travail" [des Chinois], en donnant naissance à une nouvelle population "déclassée", oblige » maintenant « l'État à intervenir, et à intervenir non plus dans les termes de la répression mais dans ceux de la discipline ${ }^{4} \cdot$

2 C'est dans ce contexte donc que la "société harmonieuse 》 (hexie shehui 和谐 社会) fait, en 2005, son entrée dans le paysage politique et discursif chinois. ${ }^{5}$ Parce la rhétorique socialiste du Parti n'est plus crédible (notamment depuis la quasi-disparition, durant les années 1990, des prestations sociales proposées par l'Etat-providence chinois), parce que les inégalités et les injustices sociales sont de plus en plus manifestes, et parce qu'il est impossible d'avouer l'inavouable (la nécessité structurelle d'une pauvreté, en l'occurrence institutionnellement entretenue, pour créer de la richesse économique) dans une société encore qualifiée de "communiste", le PCC, menacé dans son exercice exclusif du pouvoir de plus en plus questionné, se retrouve dans la nécessité de développer un autre discours, un autre programme, capable d'expliquer, de justifier et d'accompagner les crises présentes. ${ }^{6}$ Ce discours, celui de la " société harmonieuse ", n’aura pas pour intention première de convaincre, mais de fixer un cadre rhétorique dans lequel l'inacceptable polarisation sociale peut être (et devra être) acceptée. Le discours de la " société harmonieuse » s'affirme donc être un discours de crise, et la mise en place d'un tel discours correspond à ce «travail discursif des politiques » qui, pour une grande part, consiste « dans la production de slogans, de promesses et d'engagements » dans le but de renforcer « le lien de croyance et de confiance qu'ils doivent sans cesse maintenir et entretenir précisément parce que leur pouvoir est symbolique ${ }^{7}{ }^{7}$

Autour de ce concept de "société harmonieuse", c'est donc tout un programme que va articuler la rhétorique officielle, et sur lequel nous allons revenir, en réponse aux maux de la société chinoise, trop prononcés pour ne pas être maintenant officiellement reconnus. Ainsi, le discours "fondateur" de la "société harmonieuse » expose-t-il très clairement les difficultés auxquelles la Chine doit faire face en ce nouveau millénaire. 
不平衡、经济社会发展不平衡的矛盾更加突出(...)各种思想文化相互激荡,

人们受各种思想观念影响的渠道明显增多、程度明显加深, 人们思想活动的 独立性、选择性、多变性、差异性明显增强; 社会上存在的消极腐败现象以 及各类严重犯罪活动等也给社会稳定与和谐带来了严重影响, 等等. 8

\begin{abstract}
Pour synthétiser, dès maintenant et sur un plus ou moins long terme, le développement social et économique de notre pays connaîtra, sans doute, des contradictions et des problèmes encore plus complexes et plus prononcés. (...) Dans le développement socio-économique de la Chine sont également apparues de nouvelles tendances (...) qu'il nous faut maîtriser complètement. Parmi elles, distinguons ces problèmes majeurs : la pénurie des ressources naturelles et énergétiques ; (...) le développement inégal entre les villes et les campagnes, le développement inégal entre les régions et le développement inégal au niveau socio-économique [qui] sont responsables de contradictions encore plus critiques ; (...) la cohabitation et la confrontation de toutes sortes de pensées et de cultures, l'augmentation manifeste des canaux de diffusion d'idées et de concepts propres à influencer les individus à un degré de plus en plus puissant ; l'indépendance, la sélectivité, la versatilité et la divergence des activités idéologiques des individus, manifestement plus prononcées et renforcées ; l'existence au sein de la société de phénomènes négatifs et de corruption, ainsi que de toutes sortes d'activités criminelles graves, autant d'éléments qui exercent sur l'harmonie et la stabilité de la société une influence défavorable.
\end{abstract}

$4 \quad$ Animé par cet impératif de (ré)affirmer la légitimité du pouvoir du Parti, par l'urgence des questions sociale et environnementale, et par la nécessité de constituer un corpus idéologique à même de reformuler de manière favorable la perception de la réalité à défaut de pouvoir la changer, Hu Jintao va faire de la " société harmonieuse » le concept clé de sa présidence. ${ }^{9}$

5 Le propos de cet article sera de démontrer que le credo politique officiel de la République populaire de Chine, l'édification de la «société harmonieuse » (goujian hexie shehui 构建和谐社会) s'inscrit essentiellement dans un processus de discipline et de civilisation des populations pauvres et défavorisées et participe d'une naturalisation de l'ordre social établi. ${ }^{10}$

Nous verrons donc, dans un premier temps, que la «société harmonieuse » développe un discours complexe qui fait appel aux principes confucianistes de concorde et d'harmonie des inégalités, mais qui surtout légitime les disparités socio-économiques en amalgamant les dispositions naturelles de l'individu (le caractère unique de chaque être) aux dispositions sociales (les conditions de son existence au sein de la société). En étudiant ensuite le discours fondateur de la «société harmonieuse », une allocution officielle du Hu Jintao datée du 19 février 2005, nous soulignerons l'importance primordiale de la discipline morale dans la mise en place cette harmonie sociale prônée par le président chinois. Enfin, en nous attardant quelque peu sur la campagne des « Huit honneurs et des huits hontes » (barong bachi 八荣八耻), une campagne idéologique lancée en 2006 dans le cadre de la construction de la hexie shehui 和谐社会, nous confirmerons que la « société harmonieuse » vise essentiellement à discipliner et à civiliser les populations paupérisées et vulnérabilisées par les réformes économiques qui transfigurent la Chine depuis 1978, et propose finalement une réponse morale à la question sociale.

\title{
Sous-texte - Naturalisation
}




\section{confucianiste des disparités socio- économiques}

$7 \quad$ Le discours de la « société harmonieuse » n'est pas un simple énoncé : c'est un discours officiel qui articule et développe un certain nombre de notions, de concepts et autres stratégies discursives dont l'ensemble, cohérent, représente la perception officielle de la société et constitue, en soi, un véritable programme et une vision du monde. Ce discours tend à se diffuser dans les consciences et dans les imaginaires par sa sur-représentation dans l'espace public chinois et la répétition de ses énoncés. Car ce discours officiel de la "société harmonieuse » ne se construit pas uniquement sur les quelques textes officiels (écrits ou oraux) élaborés par les hauts dirigeants ou les hautes instances dirigeantes du Parti, il est également relayé et accompagné depuis 2005 par une grande quantité de textes (articles, essais, ouvrages) "para-officiels", c'est-à-dire produits par des individus ou des entités ne s'exprimant pas au nom du Parti ou de l'Etat, et diffusés par les relais de propagande que le Parti a à sa disposition : les médias et un grand nombre de maisons d'éditions. ${ }^{11}$ Toutes ces productions forment un véritable bruit discursif autour du programme de "société harmonieuse » et, filtrées par la censure (ou l'autocensure), font toutes l'apologie du concept prôné par Hu Jintao.

8 La « société harmonieuse » est un discours complexe, qui participe avant tout d'un processus de civilisation, en ce sens qu'il développe un ensemble de principes (sociétaux, moraux, relationnels) susceptibles de mener la société chinoise à un stade qui serait "supérieur", à un faîte dans une conception évolutionniste de l'histoire. Parmi les principes exposés par la «société harmonieuse », certains concernent directement le Parti ou les institutions et les entités au pouvoir : ce sont des mesures à entreprendre et des objectifs à réaliser en terme de développement économique et de protection sociale, ou des politiques à mettre en place dans l'intention de rendre la société plus "harmonieuse". Mais d'autres principes vont concerner les individus eux-mêmes : ce sont des principes moraux, destinés à reformuler les postures intellectuelles, les consciences et les états d'esprit, qui formulent que la construction d'une Chine "civilisée" passe essentiellement par la "civilisation" de ses citoyens. Ce sont des principes déterminés par le pouvoir dominant pour les individus, une coercition qui prend la forme de la moralité et qui définit le citoyen modèle d'une société harmonieuse.

9 C'est, à nos yeux, le cour et la finalité de toute l'entreprise de propagande autour de la "société harmonieuse » : la reconfiguration morale de l'individu comme réponse aux "disharmonies" de la Chine contemporaine.

Plutôt qu'un travail étymologique, nous opterons donc pour une approche transtextuelle pour mieux cerner le(s) sens et les connotations induites par le concept hexie shehui 和谐社会. Notre refus de nous aventurer dans une analyse étymologique du terme hexie 和谐 est étayé par deux raisons majeures. Tout d'abord, nous avons lu beaucoup trop d'interprétations contradictoires (et certaines farfelues) sur l'origine et la signification étymologique du terme hexie 和谐 pour pouvoir accorder un crédit à une explication plus qu'à une autre. ${ }^{12}$ Ensuite, et surtout, le sens d'un mot, et à plus forte raison d'un concept politique, ne découle pas de ses origines mais de l'usage qui en est fait dans la rhétorique et le discours dans lequel il intervient. En ce sens, et même si hexie 和谐 évoque ou fait écho à certaines notions présentes dans l'imaginaire populaire chinois, cette 
connotation se met au service d'une stratégie discursive particulière. ${ }^{13}$ Aussi, la signification que le terme hexie 和谐 revêt dans le discours de la "société harmonieuse » vient essentiellement de son emploi dans ce contexte précis. Car, comme le rappelle le sociologue Pierre-Jean Simon, «à strictement parler, un mot n'a pas de sens : il n'a, comme peuvent nous le rappeler les linguistes, que des emplois ». ${ }^{14}$

Idéologiquement, tout d'abord, le concept de "société harmonieuse socialiste », constitue avec le concept de "développement scientifique » (kexue fazhan 科学发展), l'apport théorique (et rhétorique) majeur de Hu Jintao à l'histoire idéologique du Parti communiste chinois. ${ }^{15} \mathrm{Hu}$ Jintao perpétue ainsi une "tradition" politique, honorée avant lui par Jiang Zemin et ses «trois représentations » (san ge daibiao 三个代表) et par Deng Xiaoping et sa "société de petit confort " (xiaokang shehui 小康社会), selon laquelle le leader d'une génération de dirigeants se doit de laisser sa marque dans l'histoire du "socialisme aux caractéristiques chinoises" (you zhonguo tese de shehui zhuyi 有中国特色的社会主义) ${ }^{16}$ Cette “tradition" correspond surtout à une réalité plus pragmatique : chaque nouveau dirigeant suprême se doit d'asseoir son pouvoir sur l'ensemble de l'appareil, ou du moins sur la majorité de ses membres, et cette domination s'exerce également par le biais de symboles, de discours ou de concepts "originaux", dont l'acceptation constituera et signifiera le soutien des membres de l'appareil au nouvel homme fort (mis) en place. ${ }^{17}$

Notons ensuite, à l'instar d'un certain nombre d'observateurs, que le discours de la «société harmonieuse» présente de grandes similitudes avec ce qu'on appelait dans les années 1980 les "valeurs asiatiques". ${ }^{18}$ Dans le contexte de l'époque, l'émergence de trois des "petits dragons" (Singapour, Taiwan et Corée du Sud) dans les années 1970-1980 et la performance économique de la Malaisie, appuyées sur un pouvoir autoritaire, avaient apporté la "preuve" que le développement économique n'était pas nécessairement lié à un régime démocratique, et que le contraire s'avérait également très efficace. ${ }^{19}$

Nées dans les années 1980 à Singapour et en Malaisie, conceptualisées et revendiquées par Lee Kuan Yew (Premier ministre de Singapour) et Mohamad Mahatir bin Mohamad (Premier ministre de Malaisie), les "valeurs asiatiques" correspondent à un ensemble de principes idéologiques, formalisées dans un Livre blanc en 1990, et s'affirment comme une réponse de l'Asie orientale face à "l'hégémonie des valeurs occidentales" des droits de l'homme et de la démocratie. Ces "valeurs asiatiques" sont avant toute chose des outils de domination et de contrôle, à l'instar du confucianisme : elles déterminent un cadre idéologique à même de pouvoir souder une communauté d'individus autour de l'unité et la prospérité nationales, et déterminent que la société et son développement doivent passer avant l'individu. Ainsi, les "valeurs asiatiques" promeuvent : la nation avant la communauté et la société avant l'individu; la famille comme unité de base de la société ; la considération sociale pour l'individu soutenu par sa communauté ; le consensus plutôt que les querelles; l'harmonie raciale et religieuse. ${ }^{20}$ Ces "valeurs" développent une idéologie qui justifie l'exercice d'un pouvoir autoritaire. Rien d'étonnant alors que «ceux qui mettent le plus fortement en avant ces fameuses "valeurs" ne sont ni des philosophes ni des théologiens, mais des hommes de pouvoir ». ${ }^{21}$

Le modèle de société prôné par les "valeurs asiatiques" justifie la polarisation sociale par la promesse de l'accession à la réussite et s'appuie sur l'importance de l'abnégation et du sacrifice pour le bien de la communauté et de la société. Deux décennies plus tard, Hu Jintao, devant faire face aux crises structurelles et 
sociétales que plus de vingt années de réformes et de développement économique ont engendrées, développera un cadre idéologique similaire.

Apparu d'abord de manière assez informelle dans un rapport de Jiang Zemin lors du seizième Congrès du PCC en 2002, le concept d' « édification d'une société harmonieuse socialiste 》 (goujian shehuizhuyi hexie shehui 构建社会主义和谐社 会) fait une première apparition lors du quatrième plénum du seizième Comité central du PCC, qui se tint du 16 au 19 septembre 2004. ${ }^{22}$ Mais sa véritable intronisation publique date du 19 février 2005 lorsque Hu Jintao, dans une longue allocution à l'Ecole centrale du Parti, s'adressera aux cadres du PCC et leur exposera en détail les motifs et les modalités d'édification d'une "société harmonieuse socialiste» (shehui zhuyi hexie shehui 社会主义和谐社会). La « société harmonieuse » intervient comme réponse au défi que représente pour le PCC «le classique compromis entre efficacité [économique] et équité [sociale] ». ${ }^{23}$ Comme l'expliquent les politologues Guo Baogang et Guo Sujian, « une poursuite obsédée de la croissance économique et une légitimité basée sur la performance est une posture qui (...) se retrouve éclipsée par les injustices sociales et économiques, les inégalités régionales et salariales, et les catastrophes environnementales $» .^{24}$ Le PCC a besoin de se construire une nouvelle légitimité politique articulée autour de la crise sociale et environnementale présente. La pérennité de son pouvoir est définitivement menacée s'il ne parvient pas à lier croissance économique et égalité sociale, deux éléments à la cohabitation difficile pour ne pas dire antinomique. ${ }^{25}$ Le discours de la «société harmonieuse » aura donc pour rôle d'accompagner la situation présente, en prônant l'édification d'une société qui, tout en poursuivant un développement économique raisonné et raisonnable, mettrait l'harmonie sociale au centre de ses préoccupations et de ses actions: cette nouvelle stratégie discursive constitue les « habits neufs » du président Hu Jintao. ${ }^{26}$

Mais Hu Jintao et les hauts dirigeants du PCC «ne sont clairement pas intéressés par la construction d'une utopie moderne, mais par une reconstruction culturelle via un encadrement politique. Un tel encadrement implique la mise en valeur de nouvelles idées de manière à faire référence à un cadre discursif populaire existant, et à ainsi favoriser certaines interprétations et à en décourager d'autres ». ${ }^{27}$ Cette "reconstruction culturelle» que poursuit le discours de la "société harmonieuse » correspond à son caractère civilisateur. En stipulant des conventions sociales et des postures morales particulières, le discours de la «société harmonieuse » se propose d'éduquer les individus et de leur inculquer un ensemble de dispositions "civilisées" adapté à la «situation chinoise " (guoqing 国情). Mais la hexie shehui 和谐社会, parce qu'elle fait effectivement référence à un cadre culturel existant, s'affirme être, non une invention, mais une réinvention. En rappelant dans son allocution du 19 février 2005 que «la Chine a produit tout au long de son histoire un grand nombre de pensées sur l'harmonie sociale 》(《我国历史上就产生过不少有关社会和谐的思 想»), Hu Jintao inscrit “sa” 《société harmonieuse» dans une continuité historique et dans une "sinité" qui conféreront à son discours une légitimité culturelle. ${ }^{28}$ Mentionnant des penseurs comme Confucius, Mozi, Mencius, et des projets utopiques comme le royaume des Taiping (Taiping tianguo 太平天国), Hu Jintao cite surtout la société idéale de la «grande unité »(datong 大同) décrite par Confucius dans Livre des rites ( $L i J i$ 礼记). ${ }^{29}$

《礼记·礼运》中描绘了“大道之行也, 天下为公, 选贤与能, 讲信修睦。故人 不独亲其亲, 不独子其子, 使老有所终, 壮有所用, 幼有所长, 矜、寡、

孤、独、废、疾者皆有所养”这样一种理想社会. ${ }^{30}$ 
Le Livre des rites décrit une société idéale dans laquelle "le dao était habité et la terre appartenait à tous, les hommes talentueux et vertueux [au pouvoir] inspiraient la confiance et entretenaient l'harmonie. Les hommes de cette époque ne se préoccupaient pas uniquement de leurs parents ou de leurs enfants : toutes les personnes âgées étaient prises en charge jusqu'à leurs derniers jours, aucun adulte n'était dans le besoin, les enfants étaient accompagnés dans leur croissance et leur éducation ; les veufs, les veuves, les orphelins, les personnes isolées, les handicapés et les malades étaient tous pris en charge.

L'harmonie sociale prônée dans la hexie shehui 和谐社会 fait donc référence à ce monde idéal, cet âge d'or de la "grande unité » (datong 大同), l'idéal d'un monde unique, la « philosophie d'un monde unique» (" a one world philosophy»), au même titre donc que la " société harmonieuse $»{ }^{31}$ Cette référence au lexique de Confucius poursuit deux stratégies : il permet, à l'instar de l'idéologie confucianiste, de se baser sur des concepts confucéens réinterprétés, ou sur leur simple évocation, pour formuler un nouvel ordre social ; il permet également d'inscrire ce programme dans une continuité et une légitimité historique, de lui conférer une "sinité" propre à le naturaliser. ${ }^{32}$

Le concept de "grande unité » (datong 大同) auquel se réfère Hu Jintao implique une communauté harmonieuse et une fraternité universelle, une société juste et équitable où chaque individu voit ses besoins satisfaits, où les personnes âgées et les handicapés sont pris en charge, les jeunes éduqués, les actifs employés, une société basée sur des vertus et des valeurs morales qui mettent l'homme en harmonie avec les autres, avec la société et avec la nature. Mais comme le reconnait le professeur Shi Zhongwen, l'harmonie dont parle le Livre des rites est avant tout une affaire d'éthique et de vertus individuelles. ${ }^{33} \mathrm{La}$ cohésion de l'ensemble dépend de la capacité de chacun à s'intégrer dans le tout, par son humilité et sa disponibilité pour la communauté. L'accession au stade de la « grande unité » repose donc sur la bonne volonté de l'individu, mis au service de la société.

C'est sur cette conception de l'ordre social que s'appuie le confucianisme pour lequel le concept d'égalité ou d'unité (tong 同) « repose sur la cohérence d'un système de gouvernement total qui concerne tout le monde », dans une société où la vie (ming 命) d'un individu est « la vie telle que déterminée par le destin : elle est imposée à l'homme, tout comme son nom, par les dieux » et n'est rien d'autre qu'une "mission assignée à l'homme par le Ciel en lui donnant vie, et à laquelle il doit s'adapter au mieux en accomplissant le plus consciencieusement possible son devoir au sein de la société des hommes ». ${ }^{34}$ Le confucianisme, en transformant Confucius en une figure suprême de l'autorité, mis en place tout un système de représentations, articulé autour du concept de l'unité - l'unité du monde terrestre (tianxia 天下) avec l'ordre naturel des choses (dao 道) garantie par la cohésion du corps social dévoué dans un certain nombre de conventions et à la tête duquel se trouve l'empereur -, qui assura le maintien et la pérennité du despotisme impérial sur plus de deux milles ans.

Un système de relations sociales hiérarchisé de part en part devait manifester cette unité. Il imposerait à chacun un rang et une position déterminée, lui prescrirait des devoirs spécifiques, exigerait de lui la vertu particulière qu'il fallait pour les remplir. Ce système distribuait de façon savante, à chacun selon sa place, un devoir de soumission vers le haut et un droit de domination vers le bas. Le fondement de cet édifice était le peuple (min 民) qui n'avait que le devoir de soumission. Au sommet se trouvait l'empereur (appelé souvent shang 上, le "Haut") qui n'avait que le droit de dominer. Ce système avait une vocation, universelle, catholique. Il 
impliquait que chacun était responsable, dans son rôle, de l'harmonie du Tout et portait, en ce sens, une responsabilité totale. Il en résultait un devoir généralisé d'abnégation en faveur de la totalité. Le moi était haïssable. 35

Cette conception de la société préfigure les formes de totalitarisme du vingtième siècle, associant contrôle total de la société par l'État et unité affirmée d'un peuple-Un. ${ }^{36}$ Car comme le rappelle le sinologue Wolfgang Bauer, « l'équation fondamentale pour un ordre mondial idéal, la tranquillité et l'égalité, peuvent donc déjà se trouver chez Confucius, bien que leur forme nous évoque plutôt une stabilisation uniforme de l'inégalité ».37 Nous verrons que c'est exactement ce que recherche le programme de la « société harmonieuse » de $\mathrm{Hu}$ Jintao : une stabilisation uniforme des inégalités dans l'acceptation par chacun de ses conditions d'existence (par une sorte de reformulation du concept de ming 命) et le sacrifice de chacun dans la construction d'un projet supérieur, la nation chinoise.

Nous ne tenons pas à amplifier plus qu'il ne faudrait la tonalité confucianiste de la « société harmonieuse », ne serait-ce que parce que l'utilisation de la figure de Confucius s'inscrit également dans une tentative de légitimer, de naturaliser, ce discours. Mais d'une manière générale, le programme de la "société harmonieuse », non seulement développe un ordre social très inspiré de la conception confucianiste de la société telle que nous venons de la présenter, mais également instrumentalise tout un corpus littéraire et toute une «culture au point de la refondre entièrement et d'en faire la base de l'ordre nouveau » tout comme l'ont fait auparavant « les empereurs, leurs conseillers et leurs agents » depuis la dynastie Han 汉.$^{38}$ Car comme l'explique Jean-François Billeter,

pour faire oublier la violence et l'arbitraire dont l'empire était né, et par lesquels il se soutenait, il devait paraître conforme à l'ordre des choses. (...) Tous les domaines du savoir, toute la pensée, le langage, les représentations devaient concourir à persuader les esprits que cet ordre était, dans son essence, naturel. C'était le moyen le plus efficace d'assurer la pérennité du régime impérial, de ses hiérarchies, des formes de domination qu'il imposait, de la soumission qu'elles exigeaient. De cette refonte générale est née ce que les Chinois eux-mêmes ont considéré depuis lors, et que l'on considère aujourd'hui encore, en Chine ou ailleurs, comme la civilisation chinoise. ${ }^{39}$

Le confucianisme est né de cette volonté d'asseoir une domination en la naturalisant et en l'inscrivant dans une "tradition" culturelle réinventée pour l'occasion. ${ }^{40}$ Le confucianisme sur lequel se sont basés durant des siècles l'empire et la société chinoise pour fonctionner, n'est qu'une interprétation de la pensée développée par Confucius érigée en idéologie et en système, la transformation d'une philosophie morale régulant les rapports de l'individu avec le monde et dans le monde au rang d'idéologie politique imposée sur les comportements des individus afin de réguler la société et l'ordre. ${ }^{41}$ Et c'est là tout l'intérêt : partir de l'individuel et réguler l'individu pour enrégimenter la collectivité et maintenir l'ordre social. En ce sens, la conception confucianiste de l'harmonie est finalement très explicite.

The concept of harmony (hexie) -- a classic Confucian term that connotes humanism, decency and honourable behaviour -- has been reinterpreted since imperial times by those in power to foster popular obedience and respect for authority. (...) its resurgence as the main keyword of the $\mathrm{Hu}$ government's ideology (...) follows similar interpretations of Confucian doctrine as practiced by Asian states like Singapore, where it is used to 
justify marrying authoritarian politics with capitalist prosperity. In such selective rereading of the Confucian concept of harmony, compliant behaviour and submission to authority are emphasized, whereas notions of social justice, political dissent, and the moral duty of citizens to criticize abusive or oppressive rulers, are conveniently ignored. ${ }^{42}$

Le concept d'harmonie (hexie) - un terme confucéen classique qui implique humanisme, bienséance et comportement respectable - a été réinterprété depuis l'époque impériale par ceux au pouvoir pour favoriser l'obéissance populaire et le respect de l'autorité. Sa résurgence comme mot clé de l'idéologie du gouvernement de $\mathrm{Hu}(. .$.$) s'inscrit également dans les$ interprétations de la doctrine confucéenne tels qu'elles furent mise en pratique par des États asiatiques comme Singapour, où elle fut utilisée pour justifier le mariage entre politique autoritaire et prospérité capitaliste. Cette relecture plutôt partiale du concept confucéen d'harmonie insiste sur la docilité des comportements et la soumission à l'autorité, et ignore de manière commode les notions de justice sociale, de dissidence politique et de devoir moral des citoyens à critiquer un gouvernement coupable d'abus ou d'oppression.

La « société harmonieuse » s'affirme donc comme une double réinterprétation du confucianisme et de la pensée de Confucius, une transtextualité complexe dont nous retiendrons qu'elle implique un processus de pacification et de régulation des comportements sociaux. Car «la société d'harmonie socialiste revisite en effet le passé commun de la Chine pour mieux le réinventer et enfermer l'individu dans un "pseudo-holisme" aux tentations totalitaires. Cette harmonie de l'ordre social fait taire les dissonances $» .43$

Soulignons enfin une dernière fois que cette référence directe au lexique de Confucius permet également d'ancrer immédiatement le discours de la «société harmonieuse » dans les esprits en l'inscrivant dans un héritage culturel et intellectuel valorisant et "naturalisant". Cette "confucianisation" du discours politique permet d'installer cet ensemble d'énoncés dans une certaine "sinité" tout en lui offrant un cadre idéologique et moral très efficace et favorable au maintien de l'ordre établi. Cette stratégie prend place dans une temporalité récente où Confucius a été officiellement imposé comme le nouveau "champion" de la Chine contemporaine. ${ }^{44}$ Le chercheur Sébastien Billioud confirme qu'il existe un "regain d'intérêt, très fort depuis le début des années 2000, pour la culture classique " en Chine, regain qui se manifeste sous différentes formes (religieuses, éducatives, touristiques) et qui s'illustre sur de multiples supports (feuilletons télévisés, sites internet). "La référence au "confucianisme" », que l'on « retrouve "en fragments" un peu partout » dans les productions culturelles et dans les médias, participe à la « réinvention (...) d'une culture traditionnelle », et si nous avons expliqué cette résurgence idéologiquement, on ne peut toutefois pas nier l'impact et les conséquences que l'émergence brutale de l'économie de marché ont pu avoir sur les esprits, les comportements, les pratiques et les relations humaines, le bouleversement des références et des repères qu'elle a induit chez certains et l'éventuel besoin d'une quête de sens ou d'identité incarné dans un intérêt vivace pour des pratiques et des pensées "traditionnelles". ${ }^{4}$

Mais l'utilisation officielle de concepts "hérités" de Confucius ne confère pas nécessairement au discours de la «société harmonieuse» une force de conviction, cela signifie seulement que ce discours apparaît à sa place, juste, dans le contexte de l'époque. La force de conviction, comme l'explique Pierre Bourdieu, ne vient pas du discours lui-même mais du pouvoir symbolique que possède le porteur du discours. "Ce qui fait le pouvoir des mots et des mots d'ordre, pouvoir de maintenir l'ordre ou de le subvertir, c'est la croyance dans la 
légitimité des mots et de celui qui les prononce, croyance qu'il n'appartient pas aux mots de produire », et cette croyance est justement ce qui manque au Parti depuis la répression arbitraire de Tiananmen. ${ }^{46}$ Les discours ne prennent plus car «le pouvoir symbolique comme pouvoir de constituer le donné par l'énonciation (...), ne s'exerce que s'il est reconnu, c'est-à-dire méconnu comme arbitraire », alors que l'arbitraire du Parti Communiste chinois est connu et reconnu de tous. ${ }^{47}$

Nous allons voir, par l'étude des quelques textes officiels, que le discours de la "société harmonieuse», même si un certain nombre de mesures et de dispositions sociales vont effectivement être décidées (mais pas nécessairement appliquées ni applicables, loin s'en faut), vise avant tout à plier les consciences vers une disposition et une disponibilité d'esprit favorables à un nouvel ordre social où l'abnégation de l'individu serait justifiée par la réussite du "Tout", c'està-dire la nation chinoise et son développement conçu en termes essentiellement économiques.

\section{Texte - Discours "fondateur" de la « société harmonieuse » du 19 février 2005}

La diffusion des concepts politiques ou idéologiques officiels au sein de l'espace public chinois se déroule généralement sous la forme de campagnes de masse, mobilisant l'ensemble du système de propagande du Parti. Les divers énoncés et slogans de la "société harmonieuse », initialement présentée le 19 février 2005, ont donc été projetés dans le champ social via de multiples canaux de diffusion publics contrôlés par le pouvoir: affichages urbains, reportages et émissions télévisées, articles de journaux, sessions d'étude et sites internet. Les relais dont dispose le PCC pour diffuser son expression sont nombreux et invasifs, en ce sens qu'ils exercent une véritable emprise quantitative sur les sens (la vue, l'ouie sont fortement sollicitées). Cette emprise sur les sens (visuels, auditifs) n'est cependant pas, là non plus, nécessairement garantie d'emprise sur les consciences. ${ }^{48}$ Le système de propagande politique du PCC, historiquement très élaboré, reste encore très efficace et ses ramifications sont encore très présentes, même si la mondialisation de la Chine et l'augmentation exponentielle du nombre de canaux d'information différents ont inévitablement réduit son impact, et entamé sa prédominance sur l'espace public. ${ }^{49}$ Le système de propagande chinois (xuanchuan xitong 宣传系统) agit sur l'ensemble des médias existants pouvant diffuser de l'information sur le territoire chinois. ${ }^{50}$

En plus des allocutions publiques ou des slogans politiques à proprement parler, les campagnes idéologiques sont généralement "secondées" par une "salve" d'expressions individuelles: des sociologues, des historiens, des enseignants, des intellectuels ou des journalistes, vont s'exprimer publiquement dans les médias officiels pour exprimer une position, nécessairement favorable, sur la campagne en cours, sur sa signification et sur son importance. Cette "seconde salve" est nécessaire car elle participe à la tautologie du système : des personnalités faisant autorité confirment le bien fondé de la campagne en cours (et cette absence de discorde fait sa légitimité), et cette prise de parole publique confirme en retour leur autorité en la matière. Cette tautologie renforce l'omniprésence du discours, déjà dominant, dans le champ social, et participe à 
sa légitimité. Car comme l'expliquent Pierre Bourdieu et Luc Boltanski, la circulation d'un discours fait partie intégrante de sa production et il est impossible de les dissocier : les "propriétés les plus spécifiques » du discours, "à savoir l'ensemble des présupposés admis sans examen et la croyance collective qui leur est accordée, sont produites dans et par la circulation même », et le champ de production du discours s'affirme être « le lieu d'une circulation circulaire propre à produire un effet d'autoconfirmation et d'autorenforcement, et par là l'illusion de l'évidence immédiate ${ }^{51}$

La «société harmonieuse » ne s'exprime pas que dans les textes officiels : c'est une présence publique (essentiellement visuelle) permanente que permettent tous les moyens mis à disposition par un État qui garde le contrôle du domaine public ; c'est un discours qui imprègne les expressions publiques et, dans une autre mesure, les relations privées, et qui dispose de relais (médiatiques, institutionnels, etc.) qui vont bien au-delà des simples allocutions officielles ; c'est également un discours (re)construit au fur et à mesure qu'il se propage, et qui s'appuie sur un ensemble de préconstruits sociaux et culturels, la doxa, qu'il participe à légitimer en retour. Comme l'explique le linguiste Thierry Guilbert, " un discours s'autorise à la fois de la force de légitimation que donne l'implicite du coutumier, de la tradition, de l'idéologie du quotidien, de l'opinion et du sens commun, et s'appuie sur les forces idéologiques institutionnelles ou systèmes idéologiques constitués $» .^{2}$

L'allocution de $\mathrm{Hu}$ Jintao le 19 février 2005 devant les cadres de l'Ecole centrale du Parti durant laquelle le président insiste sur la nécessité d'augmenter la capacité du Parti à édifier une "société harmonieuse socialiste » est donc la première sortie officielle du président sur le sujet, et pose ses bases idéologiques et pratiques.

Arguant qu'à partir d'un certain stade, « lorsque le Produit Intérieur Brut par personne dépasse la barre des mille dollars américains par habitant, le développement économique et social entre alors dans une phase cruciale », $\mathrm{Hu}$ Jintao souligne dans ce discours que certains pays ont négocié cette phase cruciale avec succès, "parce que des mesures appropriées ont favorisé le développement rapide de l'économie et le progrès de la société dans la stabilité », confirmant ainsi que le développement prioritaire de l'économie reste au cœur de la stratégie politique du gouvernement chinois. $^{53}$ Sur la base de cette comparaison, Hu Jintao enferme alors la Chine dans ce postulat déterministe où le salut (le « succès ») d'une nation passe par le développement de son économie et la stabilité de sa société.

Hu Jintao continue en rappelant que le Parti a pour préoccupation et pour devoir de "protéger et développer les intérêts fondamentaux de la grande majorité du peuple » chinois, et non plus du peuple chinois dans sa totalité, une distinction apparue avec le concept des trois représentations (san ge daibiao 三 个代表) développé par Jiang Zemin en 2001; et qu'il se doit de renforcer son " administration de la société, de créer un bon environnement humain, de maintenir un bon ordre social, de protéger la stabilité de la société et de garantir aux masses une vie paisible et un travail heureux », un énoncé que l'on peut juger équivoque, la protection de la stabilité sociale et le maintien de l'ordre pouvant autant passer par la prévention que par la répression. ${ }^{54}$ Mais tout cela s'avère indispensable pour permettre au Parti de mener à bien ses « trois grandes tâches historiques》(《三大历史任务») : 《réaliser la modernisation de notre pays, achever l'unité de notre patrie, protéger la paix mondiale» (《推进现代化建设、 完成祖国统一、维护世界和平》). ${ }^{55} \mathrm{La}$ notion de “modernisation” (xiandaihua 现 
代化) est une stratégie discursive habilement utilisée de manière récurrente dans le discours officiel pour justifier un certain nombre de choix économiques, aux conséquences sociales souvent problématiques, réalisés dans le cadre général du développement (fazhan 发展) du pays et qui correspondent en réalité à la mondialisation de l'espace social chinois. La notion de "modernisation" articule autour d'elle toute une rhétorique anxiogène centrée sur les termes de "retard" et de "rattrapage", une rhétorique déjà initiée sous l'ère maoïste quand il s'agissait de " dépasser l'Angleterre et de rattraper les Etats-Unis » (lors du " Grand bond en avant ", Da yuejin 大跃进). Mais présentement, la "modernisation" sert de paravent discursif pour tous les accomplissements de l'idéologie libérale dominante, elle exerce ce que Bourdieu appelle une «fonction d'écran » (ou «fonction de masques »). ${ }^{56}$ Le sociologue Jean-Marc Vernier voit dans le terme "modernisation" un "commode outil d'euphémisation politique », expliquant que « tous les gouvernants prônent ainsi résolument la nécessité de moderniser (...) l'État, les institutions. Ce bel unanimisme n'a d'égal que l'élasticité d'un mot fourre-tout qui ne répond plus qu'à des injonctions adressées au corps social et aux citoyens $\gg .57$

33 Mais, continue Hu Jintao, les conditions présentes ne sont pas si défavorables à l'harmonie sociale. Elles sont même plutôt "propices » (《有利条件»). Tout d'abord, «la direction du pays par le Parti communiste et notre système socialiste constituent les garanties les plus fondamentales pour la construction d'une société harmonieuse socialiste »; cette affirmation, fondée sur elle-même, est nécessaire pour renforcer et légitimer la présence du Parti communiste à la tête du pays..$^{58}$ Ensuite, le «développement continu depuis la fondation de la Nouvelle Chine et plus particulièrement depuis les réformes et l'ouverture » ont renforcé la «puissance nationale » (guoli 国力), et l'unité de la nation est assurée puisque « toutes les classes, tous les partis politiques, toutes les ethnies et toutes les communautés jouissent politiquement d'un statut égal, leurs intérêts fondamentaux sont identiques ».59

Néanmoins, et la remarque est d'importance, Hu Jintao reconnaît que «la Chine se trouve toujours et se trouvera encore pendant une longue période dans la phase initiale du socialisme» et que «l'établissement d'une société harmonieuse socialiste est une tâche difficile qu'il nous faudra assumer avec responsabilité sur une longue période. De la même manière que la construction d'un pays socialiste modernisé représente un long processus historique, la construction d'une société harmonieuse socialiste s'avère également être un long processus historique qu'il faudra sans cesse encourager $» .^{60}$ Nous soulignons ce point particulier, car le Parti communiste chinois n'a eu de cesse de justifier l'échec de certains objectifs sociaux majeurs qu'il s'était fixé par la complexité du processus et des opérations, par la nécessité d'un long terme et l'importance de la patience et de l'abnégation. ${ }^{61}$ La prudence rhétorique de Hu Jintao soulignant que la Chine restera encore longtemps dans la phase initiale du socialisme, et qu'il faudra également redoubler d'efforts sur une longue période indéterminée pour construire une "société harmonieuse », révèle non seulement l'ampleur et la difficulté effectives de la tâche, mais également les limites et les inerties inhérentes à la situation sociale et politique chinoises (en tout premier lieu desquelles l'exercice exclusif du pouvoir par une seule entité, quand bien même hétérogène, et les collusions d'intérêts qui en découlent).

Hu Jintao en vient alors aux modalités pratiques de mise en œuvre de la «société harmonieuse ». Insistant sur l'importance de prendre pour principes directeurs «la théorie de Deng Xiaoping et l'importante pensée des "trois 
représentations” 》 (《邓小平理论和“三个代表”重要思想》), une “formalité” discursive obligée pour inscrire le discours de la «société harmonieuse » dans une continuité idéologique, le texte insiste sur l'importance fondamentale de 《maintenir comme objectif central le développement de l'économie 》 (《坚持以 经济建设为中心》) tout en mettant en pratique le «concept de développement scientifique» (《科学发展观》), c'est-à-dire un développement durable et rationnel. ${ }^{62} \mathrm{Hu}$ Jintao appelle à «placer l'homme au centre des choses » («以人 为本»), et à "prendre les intérêts fondamentaux de la grande majorité du peuple chinois comme points de départ et points d'achoppement fondamentaux du travail réalisé par l'État et le Parti ${ }^{63}$ Ce concept de « grande majorité du peuple chinois ", introduit par les "trois représentations" de Jiang Zemin, diffère sensiblement du credo initial d'un Parti s'affirmant « au service du peuple » (wei renmin fuwu 为人民服务) dans sa totalité. Le Parti réaffirme qu'il est désormais le parti d'une partie de la population qu'il favorise par un accès privilégié à la société de consommation. ${ }^{64}$ Ce concept permet également de demander à l'individu de sacrifier ses intérêts "individuels" au nom des intérêts d'une "grande majorité du peuple chinois" dont il ne ferait pas nécessairement partie. Hu Jintao appelle également le Parti à accorder toute son « attention à l'égalité sociale » (《必须注重社会公平》), puis résume en une phrase les impératifs du gouvernement chinois pour les années à venir :

\title{
必须正确处理改革发展稳定的关系, 坚持把改革的力度、发展的速度和社会 可以承受的程度统一起来, 使改革发展稳定相互协调、相互促进, 确保人民 群众安居乐业, 确保社会政治稳定和国家长治久安. 65
}

\begin{abstract}
Il est indispensable de traiter correctement la relation entre réformes, développement et stabilité, de persévérer dans cette approche unifiée réunissant intensité des réformes, vitesse du développement, et capacité de tolérance de la société de manière à coordonner et à promouvoir mutuellement réformes, développement et stabilité, de garantir aux masses populaires une vie paisible et un travail heureux, de garantir la stabilité politique et sociale ainsi que la gouvernance à long terme et sans trouble du pays.
\end{abstract}

Ce propos nous parait essentiel, car il révèle encore une fois que la priorité est au développement économique de la Chine, auquel il faudra habituer et adapter la population chinoise. Répétons tout de même que cette stratégie n'a rien de particulier à la Chine ni au Parti communiste chinois. Comme le disait Jean-François Billeter, «la société dans son ensemble, en particulier la société urbaine et le régime lui-même, n'ont fondamentalement plus d'autre perspective qu'un développement conçu en termes purement économiques. L'imaginaire dominant est désormais le même qu'ailleurs ». ${ }^{66}$

$\mathrm{Hu}$ Jintao poursuit son allocution en spécifiant les dix tâches à accomplir, qui s'imposent au Parti et à l'ensemble de la société chinoise, pour " promouvoir la construction d'une société harmonieuse socialiste 》(《促进社会主义和谐社会建 设»). ${ }^{67}$ En tout premier lieu, il faut «maintenir consciencieusement le développement continu, rapide, coordonné et sain de l'économie ", précisant une nouvelle fois que "la résolution des nombreux problèmes et contradictions auxquels fait face la Chine (...) réside dans le développement $»{ }^{68}$ Le président Hu Jintao souligne ensuite l'importance du développement économique pour créer un 《meilleur système de sécurité sociale ( «更完善的(...)社会保障体系》), " créer davantage d'opportunités professionnelles et satisfaire continuellement les divers besoins des masses populaires », affirmant qu'un développement économique «cordonné et sain (...) est la garantie majeure, pour les masses, 
d'une amélioration continue de leur niveau de vie ». ${ }^{69}$ Abordant la question paysanne, Hu Jintao assure que « la vie des nombreux paysans s'améliore de jour en jour, leur qualité s'améliore également, les nombreux villages paysans vivent maintenant dans un climat paisible et bienveillant ", une vision que tout le monde ne partage pas nécessairement sur le terrain. ${ }^{70}$ Evoquant ensuite l'importance de " développer consciencieusement la démocratie socialiste » («切) 实发展社会主义民主》) et d'appliquer 《les principes fondamentaux d'un État de droit » (《切实落实依法治国的基本方略》), l'allocution aborde enfin la question de la morale. ${ }^{71}$ Il faut « renforcer consciencieusement la construction d'une morale idéologique 》 (《切实加强思想道德建设》) réaffirme $\mathrm{Hu}$ Jintao, soutenant que "l'harmonie d'une société et la capacité d'un pays à installer un gouvernement sur un long terme et sans troubles dépendent en grande partie de la qualité morale de l'ensemble de ses membres $" .^{72}$ Il faut donc éduquer le peuple de manière à susciter chez lui « une confiance inébranlable dans le socialisme aux caractéristiques chinoises » et une « fibre nationale centrée sur le patriotisme » tout en le formant à « l'esprit de notre temps centré sur les réformes ».73 Pour ce faire, Hu Jintao appelle à mettre en œuvre énergiquement "les travaux de construction d'une morale citoyenne » (《要积极实施公民道德建设工程》). ${ }^{74}$

\title{
广泛开展社会公德、职业道德、家庭美德教育，在全社会倡导爱国守法、明 礼诚信、团结友善、勤俭自强、敬业奉献的基本道德规范, 培养良好的道德 品质和文明风尚.75
}

\begin{abstract}
Il faut développer massivement une morale publique au sein de la société, une éthique professionnelle, et une excellence morale au sein des foyers, promouvoir dans toute la société des normes morales élémentaires basées sur l'amour de la patrie et le respect de la loi, la politesse, les bonnes manières, l'honnêteté et la confiance, la cohésion et la fraternité, la diligence, l'abnégation et l'amélioration de soi, le respect du travail et le sens du sacrifice. Il faut cultiver de bonnes qualités morales et des mœurs et coutumes civilisées.
\end{abstract}

《Morale ( (daode 道德) et 《civilisation » (wenming 文明) sont des éléments clés de la "société harmonieuse ", dont le discours dessine les contours d'un "homme nouveau" : un "citoyen civilisé" et patriote, c'est-à-dire respectueux du Parti et de ses politiques, et à la "qualité morale" élevée, c'est-à-dire capable de faire passer les intérêts de la société, tels que le Parti les définit, avant ses propres intérêts, avec «abnégation 》 (qinjian 勤俭) 《sens du sacrifice» (fengxian 奉献) en faveur de la « cohésion et de la fraternité » (tuanjie youshan 团结友善) de la société. ${ }^{76}$ Car, comme l'explique l'historien Limor Yagil, «l'idée de construire un homme nouveau, plus précisément un nouvel être humain, est inhérente à tout programme utopique d'une part, et constitue d'autre part le but et le noyau de toute idéologie totalitaire ou autoritaire ». Cet homo harmonicus, cet "homme de la société harmonieuse", est au cœur du discours de la « société harmonieuse », il est l'élément primordial et la condition sine qua non à l'harmonie sociale. C'est essentiellement en ce sens que le discours de la « société harmonieuse » s'inscrit dans ce processus global de civilisation de la population chinoise, c'est-à-dire dans ce projet de faire des Chinois (et en particulier les plus défavorisés et les plus exploités d'entre eux) des citoyens "polis" et "civilisés", capables de se mettre sans protester au service de l'économie de marché et de son fonctionnement. ${ }^{77}$ Et quand $\mathrm{Hu}$ Jintao insiste pour «donner une place prioritaire à l'éducation» (《把教育摆在优先地位》), 《garantir l'égalité dans l'accès à l'éducation 》 (《保障教育公平》), et 《bâtir un système éducatif sain et solide» (《构建健全的教育体系》) de manière à 《promouvoir sans cesse 
l'augmentation et l'amélioration de la qualité de l'ensemble de la nation » (《促进 全民族素质不断提高》), il confirme le propos de Michel Foucault quand celui-ci précise que « tout système d'éducation est une manière politique de maintenir ou de modifier l'appropriation des discours, avec les savoirs et les pouvoirs qu'ils emportent avec eux $» .^{78}$

Le discours de la "société harmonieuse", au travers de cette allocution officielle de $\mathrm{Hu}$ Jintao, constitue donc un programme qui se veut totalisant en "visant l'ensemble de la collectivité » et qui cherche à «jouer un rôle de "cadrage" » en prétendant " fixer le cadre général où lieux communs et énoncés orthodoxes peuvent être énoncés ", aspirant par là à "un rôle normatif de délimitation des représentations de la collectivité ». ${ }^{79}$ Mais la «société harmonieuse » telle que la présente Hu Jintao ressemble plus à une utopie, au sens propre et au sens figuré: au sens propre, parce que la "société harmonieuse » se présente comme cette société de nulle part puisque encore à construire, une "société politique» caractérisée « par une constitution rationnelle, un idéal de perfection absolue, de la réalisation duquel découle le bonheur de tous les citoyens »; au sens figuré, parce que les énoncés se résument pour la plupart à des objectifs à atteindre, des intentions et des impératifs officiels que rien dans la situation chinoise actuelle ne semble particulièrement conforter, et dont la réalisation semble au mieux difficile, si ce n'est impossible, dans les conditions présentes. S'il n'y a aucun doute à ce que le gouvernement chinois continue une politique centrée sur la croissance économique, il est par contre raisonnable de douter de sa capacité (et plus simplement de la possibilité) à mettre en place, par exemple, un système de sécurité sociale universel et efficace, un besoin pourtant urgent tant en zones urbaines qu'en zones rurales.

Finalement, à étudier de près cette allocution et les énoncés qu'elle développe, on peut estimer que, mis à part le développement économique et le développement d'un arsenal sécuritaire qui semblent être des objectifs réalisables pour le pouvoir en place, la construction d'une "société harmonieuse » repose manifestement et principalement sur la capacité du "citoyen" chinois à se comporter de manière "civilisée", à faire preuve d'une haute "qualité morale" et à consacrer efforts et sacrifices au développement de l'économie et à la construction d'une nation chinoise unie et "harmonieuse". La "société harmonieuse » s'affirme donc comme une vision du monde, elle met en place un modèle civilisationnel au sein duquel l'homo harmonicus joue un rôle fondamental : elle n'est donc pas uniquement un programme politique, elle est également, voire essentiellement, affaire de perception du monde, de posture intellectuelle, un processus de configuration morale des individus centré sur l'abnégation, la patience, le sacrifice et le dévouement à la nation que la campagne des « Huit honneurs et huit hontes » va par la suite exalter. ${ }^{80}$

\section{Para-texte - La campagne d'éducation civique des « Huit honneurs et huit hontes »}

41 Les « Huit honneurs et huit hontes 》 (ba rong ba chi 八荣八耻), développés par Hu Jintao le 4 mars 2006 lors d'une allocution à la Conférence consultative politique du peuple chinois, sont huit préceptes construits de manière parallèle (chaque précepte est constitué de deux parties de sept caractères chacune) censés 
exprimer et signifier la «conception socialiste de l'honneur et de la honte » (shehuizhuyi rongruguan 社会主义荣辱观). ${ }^{81}$ La construction de ces préceptes comme des versets, avec deux structures de sept caractères chacun, permet une mémorisation plus "ludique" car la forme, symétrique, permet la récitation; et la récitation a ceci de particulier qu'elle permet l'énonciation et la diffusion d'un contenu distancié de son sens. La récitation permet une meilleure imprégnation des consciences car elle ne passe pas (entièrement) par une assimilation du sens mais par une assimilation du son.

Chaque précepte constitue un énoncé qui prétend distinguer le bien du mal, le glorieux du honteux, et expose une posture morale qu'il serait un "honneur" d'adopter et une "honte" de ne pas respecter. Ce code moral, destiné à l'origine à moraliser la vie politique, se retrouva très rapidement et massivement exposé publiquement, s'avérant finalement être une vaste entreprise d'éducation morale à destination de l'ensemble de la population. ${ }^{82}$

Dans cette allocution, Hu Jintao inscrit encore une fois son concept (la " conception socialiste de l'honneur et de la honte ») dans une continuité et une cohérence idéologiques lorsqu'il signifie que «la construction d'une société de petit confort sur tous les plans, et l'accélération du processus de modernisation socialiste nous impose de mettre le développement d'une culture socialiste avancée parmi nos tâches les plus prioritaires ». ${ }^{83}$ Ce terme, "culture socialiste avancée » (shehuizhuyi xianjin wenhua 社会主义先进文化), que nous retrouvons dans le «Programme de mise en œuvre de la construction d'une morale citoyenne » ne doit pas s'interpréter de manière trop étroite et doit être compris dans un sens anthropologique, faisant référence de manière globale aux mœurs et au mode de vie des individus. ${ }^{84}$ Ainsi, il apparaît impératif à Hu Jintao de développer une culture socialiste, et pour se faire de « concentrer notre attention sur l'élévation de la qualité des individus》 (《着眼于提高人的素质》), de 《renforcer l'édification morale et idéologique» (《加强思想道德建设》) de manière à «former des citoyens socialistes porteurs d'idéaux, de morale, de culture et de discipline $» .^{85}$ Le texte revient alors sur cette notion, récurrente, de “qualité" (suzhi 素质) de la population qu'il faudrait élever en affirmant que « la compétition globale entre les grandes puissances de ce monde est en finalité une compétition qui se joue sur la qualité de la nation », nation à prendre au sens de la communauté imaginée d'individus, ce qui revient à stigmatiser ceux qui parmi la population seraient de moins bonne "qualité", les moins "civilisés", culturellement les moins "avancés", "responsables" proclamés du "retard" de la Chine dans la compétition mondiale. ${ }^{86}$ Ce type de discours est le discours typique de l'État néo-darwiniste tel que le définit le sociologue Loïc Wacquant, à savoir un État qui

érige la compétition en fétiche et célèbre à tout crin la " responsabilité individuelle », qui «se replie alors sur ses fonctions régaliennes de maintien de l'ordre, (...) ainsi que sur sa mission symbolique de réaffirmation des valeurs communes par la vitupération publique des catégories déviantes. Ce darwinisme nouvelle manière loue et récompense les "gagnants" pour leur vigueur et leur intelligence et fustige les "perdants" de la "lutte pour l'existence" économique en pointant leurs carences de caractère ou de comportement. ${ }^{87}$ défavorisées par les choix économiques et politiques du Parti (les mingong, les xiagang, les chômeurs et les travailleurs pauvres) mais également tous les individus en situation de contestation ou de rupture avec le discours officiel du 
développement : ce sont ces populations qu'il s'agira de discipliner et d'éduquer, ce qui est d'ailleurs le propos principal des « Huit honneurs et huit hontes », et dont il s'agira de parfaire "l'édification morale et idéologique ». ${ }^{88}$ Toutes ces notions : développement, "modernisation", "qualité", réformes, opposés au "retard" et à la "médiocrité", constituent un enchevêtrement discursif et sont le lexique d'un processus de civilisation de la population chinoise.

$\mathrm{Hu}$ Jintao continue en soulignant le "rôle fondamental de l'éducation dans l'élévation de la qualité d'un individu » (《教育对提高人的素质的基础性作用》) et appelle à réformer l'éducation nationale pour permettre la " généralisation de la scolarisation obligatoire » (《加大对义务教育(...)的投入»), dont il faut rappeler qu'elle est, en Chine comme ailleurs, le principal médium des discours officiels et le lieu où se joue l'éducation civique, et où se construit "l'identité nationale". ${ }^{89}$ Pour mettre en place cette «culture avancée », Hu Jintao insiste pour que la Chine « forme et cultive des individus talentueux en grand nombre» (《培养大批 优秀人才») car 《le prestige d'une nation, vient avant tout de ses talents 》 (《国家 兴盛, 人才为本»). ${ }^{90}$ Cette notion de "talent", à rapprocher de celle de "qualité", est encore et avant tout une perception officielle (ce qui n'est du reste pas l'apanage de la Chine), et le processus est tautologique: sera défini «talentueux » ce qui sera conforme à la «culture socialiste avancée » et à la «morale citoyenne » préalablement définies..$^{91}$

$\mathrm{Hu}$ Jintao évoque enfin l'importance d'instituer «de bonnes mœurs sociales $» .^{92}$

三是要树立良好的社会风气。社会风气是社会文明程度的重要标志(...)。树
立良好的社会风气是广大人群众的强烈愿望, 也是经济社会顺利发展的必
然要求。在我们的社会主义社会里, 是非、善恶、美丑的界限绝对不能混
淆, 坚持什么、反对什么, 倡导什么、抵制什么, 都必须旗帜鲜明.93
Les conduites sociales sont un indicateur du degré de civilisation d'une
société (...). Instituer de bonnes mœurs sociales est le souhait le plus cher
des larges masses populaires, c'est aussi une condition indispensable au
bon fonctionnement de l'économie et de la société. Dans notre société
socialiste, la frontière entre le vrai et le faux, le bien et le mal, le beau et le
laid ne peut pas être floue. Il est impératif de bien distinguer ce à quoi
s'opposer, ce dans quoi il faut persévérer, ce qu'il faut promouvoir et ce à
quoi il faut résister.

Hu Jintao affirme donc l'importance d'une moralité socialiste intègre pour que chaque citoyen puisse distinguer le bien du mal. La nécessité d'un cadre moral s'impose dans l'idée de discipliner et de "civiliser" la population chinoise : «il faut éduquer les cadres et les masses populaires, et en particulier les jeunes, à la conception socialiste de l'honneur et de la honte » conclut-il..$^{94}$

Le texte termine donc par l'énoncé des huit honneurs et des huit hontes :

以热爱祖国为荣、以危害祖国为耻;

以服务人民为荣、以背离人民为耻;

以崇尚科学为荣、以患昧无知为耻;

以辛勤劳动为荣、以好逸恶劳为耻;

以团结互助为荣、以损人利己为耻;

以诚实守信为荣、以见利忘义为耻;

以遵纪守法为荣、以违法乱纪为耻;

以艰苦奋斗为荣、以骄奢淫逸为耻. 95

Aimer sa patrie avec ardeur est un honneur, porter préjudice à sa patrie est une honte ;

servir le peuple est un honneur, se détourner du peuple est une honte ; prôner et préconiser la science est un honneur, l'inculture et l'ignorance 
sont une honte ;

l'âpreté à la tâche et le travail laborieux sont un honneur, le loisir et l'oisiveté sont une honte ;

l'union et l'entraide sont un honneur, la recherche de son propre intérêt au détriment d'autrui est une honte ;

l'honnêteté et la loyauté sont un honneur, l'absence de scrupules et l'appât du gain sont une honte ;

observer la discipline et respecter la loi est un honneur, enfreindre la loi et transgresser la discipline est une honte ;

une vie faite d'abnégation et de labeur est un honneur, une vie faite

d'arrogance, de luxe, de débauche et d'oisiveté est une honte.

A l'énoncé de ces préceptes, il est difficile dans un premier temps de ne pas souligner que honnir «l'appât du gain » est quelque peu paradoxal quand le profit, «l'absence de scrupules» et la «recherche de son propre intérêt au détriment d'autrui » forment des pratiques intimement liées au fonctionnement d'une économie de marché, au sein de laquelle il n'existe de concurrence loyale que de mythe. ${ }^{96}$ Le développement de l'économie chinoise est passé et passe notamment, comme ailleurs, par des pratiques que les « Huit honneurs et huit hontes » (ba rong ba chi 八荣八耻) qualifieraient de “honteuses". ${ }^{97}$

Remarquons ensuite que bannir toute conduite immorale en proscrivant « le luxe, la débauche et l'oisiveté » est paradoxal quand, par ailleurs, ceux-ci sont sublimés par la société de consommation et la publicité marchande et sont le symbole d'une certaine réussite sociale, dispendieuse et ostensible. Louer "l'abnégation et le labeur » permet donc de mettre certains individus (ceux à qui le luxe est refusé) dans un cadre propice à la production marchande et au développement de l'économie en valorisant le travail salarié. C'est une stratégie discursive destinée à mettre les pauvres au travail, à « faire travailler les pauvres, là où l'illusion a déçu, et où la force s'est défaite $» 9^{9}$ Car le luxe et les modèles d'existence qui y sont associés jouent un rôle majeur dans l'acte d'achat des classes aux revenus supérieurs, et il apparaît alors manifeste que les ba rong ba chi ne s'adressent pas à l'ensemble de la population, mais essentiellement à celle défavorisée par les réformes, celle qu'il s'agira de mettre au travail. De la même manière, il conviendra de répudier le " loisir ", pourtant majoritairement induit dans la consommation de marchandises, et de louer l'âpreté à la tâche, de manière à conforter les nombreux exclus de la "société de loisirs" dans leur existence laborieuse.

Ce code moral est certes destiné à configurer les postures sociales des individus, à les discipliner, c'est-à-dire à « jauger les individus "en vérité" » sans plus aucune autre nuance que le glorieux et le honteux. ${ }^{99}$ Mais c'est également et essentiellement une stratégie discursive destinée à "civiliser" une certaine partie de la population chinoise, celle défavorisée par les réformes, c'est-à-dire à l'adapter à «l'esprit de notre temps » (shidai jingshen 时代精神), aux nouvelles donnes de l'économie de marché, pour la grandeur de la patrie, à laquelle il serait une honte de "porter préjudice». Les ba rong ba chi 八荣八耻 encadrent et normalisent par le discours les très nombreuses situations de précarité sociale et économique que subit une grande partie de la société chinoise et dont se nourrit son économie. ${ }^{100}$ Derrière leur naïveté apparente, ces énoncés forment l'architecture spirituelle de cet "homme nouveau" dont la «société harmonieuse " a besoin. ${ }^{101}$ La finalité, maintes fois exprimée, est de former des citoyens "civilisés", capables de construire, par leurs attitudes et leur perception du monde, cette harmonie sociale que le seul matérialisme historique ne peut plus produire. ${ }^{102}$ 
Le discours de la "société harmonieuse " s'inscrit donc dans un processus global de rectification morale de la population chinoise pauvre ou défavorisée de manière à faire accepter l'inacceptable (l'indispensable exploitation d'une population pauvre à entretenir) et à rendre égal l'inégal (chacun est égal devant les inégalités, en ce sens que chacun participe avec ses "qualités" au "développement" de la Chine). Un nouvel ordre social harmonieux se dessine dans les discours et les représentations, où le sacrifice individuel d'une grande partie de la population serait considéré comme normal dans une société où les inégalités évolueraient en harmonie, car indiscutables et indiscutées. Le processus de civilisation de la population chinoise, par le biais de complexes discursifs et idéologiques tels que la "société harmonieuse " sert donc à asseoir la domination d'un ordre social établi, et plus particulièrement à garantir la production et la consommation de marchandises.

\section{Notes}

1 Pierre Bourdieu, Langage et pouvoir symbolique, Paris, Seuil, 2001, p.327.

$2 \mathrm{Hu}$ Jintao 胡锦涛 (1942 - ) est depuis le 15 mars 2003 le président de la République populaire de Chine (il est le secrétaire général du Parti communiste chinois depuis 2002). Jiang Zemin 江泽民 (1926 - ) fut le secrétaire général du Parti communiste chinois entre 1989 et 2002, et président de la République populaire de Chine de 1993 à 2003.

3 Deng Xiaoping 邓小平 (1904 - 1997), “leader” de la seconde génération de dirigeants chinois (il est d'ailleurs surnommé le " petit timonier », en référence au " grand timonier » Mao Zedong), eut un rôle majeur dans l'histoire politique chinoise, bien qu'il n'occupa officiellement aucun poste majeur (il fut uniquement secrétaire général du Parti communiste chinois de 1956 à 1967, date à laquelle il fut évincé du pouvoir car jugé trop libéral). Réhabilité en 1977 au sein du Bureau politique du Comité central, l'instance dirigeante du Parti en Chine, après sa disgrâce lors de la " Révolution culturelle », Deng Xiaoping sera le véritable homme fort du pouvoir durant les années 1980. Instigateur des politiques de "réformes et d'ouverture " (gaige kaifang 改革开放) économiques de la Chine, favorable à la répression du mouvement de la place Tiananmen, Deng est le véritable dirigeant de la Chine de la fin des années 1970 jusqu'au début des années 1990.

4 Jean-Louis Rocca, La Condition chinoise : la mise au travail capitaliste à l'âge des réformes (1978-2004), Paris, Karthala, 2006, p.107. Les éléments entre crochets ont été rajoutés par nous.

5 Le terme chinois hexie 和谐 signifie se traduit généralement par harmonie ou concorde.

6 Sur la crise sociale en Chine contemporaine, voir Sun Liping 孙立平, Duanlie : ershi shiji jiushi niandai yilai de zhongguo shehui 断裂:20世纪90年代以来的中国社会 (Fracture : la société chinoise depuis les années 1990), Pékin 北京, Shehui kexue wenxian chubanshe 社会科学文献出版社, 2003. Sur la crise sociétale, voir Jean-François Billeter, Chine trois fois muette, Paris, Allia, 2006. Sur la crise environnementale, lire par exemple Brice Pedroletti, «Le revers de la médaille d'une croissance galopante », Le Monde, édition du 10 juillet 2007, Supplément Economie p. II ; Richard McGregor, " 750 ooo a year killed by Chinese pollution », Financial Times, édition du 2 juillet 2007; Joseph Kahn \& Jim Yardley " The dark side of China' boom », Herald Tribune, édition du 27 août 2007. Sur l'aggravation du mécontentement et des mouvements de protestation populaires, voir Lucien Bianco, Peasants Without the Party: Grass-roots Movements in Twentieth-Century China, New York, M.E. Sharpe, 2001; William Hurst, « Mass Frames and Worker Protest ", in Kevin J. O'Brien (Dir.), Popular Protest in China, Cambridge, Havard University Press, 2008, pp.71-87; Kevin J. O’Brien \& Li Lianjiang, Rightful Resistance in Rural China, New York, Cambridge University Press, 2006.

7 John B. Thompson, «Préface », in Pierre Bourdieu, Langage et pouvoir symbolique, Paris, Seuil, 2001, p.46.

8 Extrait de l'«Allocution du président Hu Jintao lors du séminaire organisé pour les hauts dirigeants et les cadres provinciaux autour de la thématique "augmenter notre capacité à construire une société harmonieuse socialiste” 》(《胡锦涛在省部级主要领导干 部提高构建社会主义和谐社会能力专题研讨班上的讲话》), prononcée le 19 février 2005 à l'Ecole centrale du Parti. Pour le texte intégral de cette allocution, voir Hongqi chubanshe 
红旗出版社, Xuexi hujintao zai sheng buji zhuyao lingdao ganbu tigao goujian shehui zhuyi hexie shehui nengli zhuanti yantaoban shang de jianghua daodu / ganbu qunzhong guanxin de tongsu 学习胡锦涛在省部级主要领导干部提高构建社会主义和谐社 会能力专题研讨班上的讲话导读/干部群众关心的通俗, Pékin 北京, hongqi chubanshe 红旗 出版社, 2005. Ce texte est par ailleurs consultable dans son intégralité sur le portail officiel de la République populaire de Chine sur l'Internet, le site Zhongguowang 中国网, dirigé par le Bureau de l'information du Conseil d'État (guowuyuan xinwenban 国务院新 闻办), voir $\mathrm{Hu}$ Jintao 胡锦涛, «Hu Jintao guanyu goujian shehuizhuyi hexie shehui jianghua quanwen 胡锦涛关于构建社会主义和谐社会讲话全文》, Zhongguowang 中国网, publié le 26 juin 2005, consulté le 12 août 2009 sur http://www.china.com.cn/chinese /news/899546.htm.

9 Avec celui de « développement scientifique » (kexue fazhan 科学发展).

10 Nous comprenons le terme «civilisation» (et ses dérivés «civilisé(e)(s)» et « civilisateur ») dans le sens défini par le sociologue Norbert Elias, pour qui la civilisation n'est pas seulement un état mais également « un processus qu'il s'agit de promouvoir ». (voir Norbert Elias, La Civilisation des moeurs, trad. de l'allemand par Pierre Kamnitzer, Paris, Calman-Lévy, 1991, p.69). La civilisation des mœurs dont parle Norbert Elias s'inscrit, dans la formation des États-nations modernes, comme une uniformisation et une normalisation des comportements, des habitudes et des attitudes en société, à l'aune des conventions sociales pratiquées par les classes dominantes et les élites (pratiques qui avaient d'ailleurs pour principe d'opérer une distinction d'avec les masses populaires). La civilisation des mœurs est avant tout un acte de domination et s'affirme donc comme l'incarnation du rapport dominant-dominé, c'est-à-dire un rapport qui prend chair, qui s'installe dans le corps. Sous sa forme actuelle en Chine, c'est-à-dire comme stratégie discursive et coercitive imposée sur les individus et destinée à favoriser le fonctionnement d'une économie de marché, le processus de civilisation de la population chinoise date de la fin des années 1970, avec le projet de « civilisation spirituelle » (jingshen wenming 精神文 明) établi par Deng Xiaoping (voir Nicholas Dynon, "Four Civilisations" and the Evolution of Post-Mao Chinese Socialist Ideology ", The China Journal, n ${ }^{\circ} 60$, juillet 2008). Mais cette volonté de "civiliser" et de discipliner le "bas peuple" à l'aune des mœurs et des normes pratiquées par l'élite intellectuelle se retrouve déjà dans le processus plus général de construction de la nation chinoise entamé au début du vingtième siècle.

11 Sur le système de propagande du PCC, lire la très complète étude de Anne-Marie Brady, Marketing Dictatorship: Propaganda and Thought work in contemporary China, Lanham, Rowman \& Littlefield, 2008 ; voir également Florence Padovani, L'action du département central de la propagande durant les années 1980 , thèse de doctorat soutenue à l'École des hautes études en sciences sociales (EHESS), 1996. Sur le rôle joué par les médias dans le système de propagande politique, et d'une manière plus générale, sur la complexe relation triangulaire entre l'État, les médias et le public, voir le travail (certes un peu daté) de Kevin Latham, " Nothing but the Truth: News Media, Power and Hegemony in South China », The China Quarterly, ${ }^{\circ}{ }^{163}$, septembre 2000, pp. 633-654.

12 Mentionnons tout de même cette explication originale à défaut d'être réellement crédible, qui voit dans le terme hexie 和谐, un idéal de société “démocratique" où les individus (l'élément $\square \mathrm{du}$ caractère he 和 est porteur d'une charge sémantique: « la bouche », et par extension, "l'individu ») mangeraient à leur faim (禾, " céréale »), et où l'ensemble de la population (l'élément 皆 du caractère xie 谐 est porteur de la charge sémantique : «tous») jouirait de la liberté d'expression (言, « parole », la forme d'écriture traditionnelle de xie 谐 s'écrit 諧).

13 Un exemple récent nous a été donné par l'utilisation de la figure de Jean Jaurès par Nicolas Sarkozy dans une allocution publique durant la campagne électorale de 2007 (au sujet de la stratégie électorale de Nicolas Sarkozy, voir Alain Badiou, De quoi Sarkozy est-il le nom ?, Paris, Lignes, 2007, pp.7-25). Cette utilisation ne signifie aucunement que Nicolas Sarkozy rejoint ou se retrouve dans les idées politiques développées par le socialiste Jean Jaurès : elle signifie que Nicolas Sarkozy utilise les connotations politiques et historiques associées au personnage de Jean Jaurès pour s'inscrire, lui, en tant qu'homme politique, dans une continuité historique et nationale auto-proclamée.

14 Pierre-Jean Simon, Pour une sociologie des relations interethniques et des minorités, Rennes, Presses Universitaires de Rennes, 2006, p.26.

15 Le concept de «développement scientifique » constitue en quelque sorte le volet économique de la rhétorique de Hu Jintao (la « société harmonieuse » correspond plutôt au volet social). Le "développement scientifique », proche du "développement durable", prône un développement de l'économie de manière rationalisée et raisonnée ("scientifique") par opposition à une croissance aveugle et irrespectueuse des ressources naturelles et humaines. Il intervient alors que la pollution de l'environnement devient un 
problème majeur et critique pour la population chinoise, mais surtout pour la production de marchandises elle-même. Le développement scientifique, parce que respectueux des forces en présence, est censé favoriser l'éclosion d'une société harmonieuse.

$16 \mathrm{Hu}$ Jintao fait partie de la quatrième génération de dirigeants. Sur les «trois représentations », voir Jiang Zemin 江泽民, Jiang Zemin wenxuan disanjuan 江泽民文选 第三卷 (Écrits choisis de Jiang Zemin, Volume 3), Pékin 北京, Renmin chubanshe 人民出 版社, 2006; voir également Emilie Tran, "Les "trois représentations" », in Thierry Sanjuan (dir.), Dictionnaire de la Chine Contemporaine, Paris, Armand Colin, 2006, p. 260. Inscrite dans la constitution de la République populaire de Chine, la théorie des « trois représentations » stipule que le Parti communiste chinois se doit de représenter et d'incarner « les exigences de développement des forces productives avancées de la société chinoise 》 (《中国先进社会生产力的发展要求》), 《le dynamisme de la culture avancée chinoise » (《中国先进文化的前进方向》), et 《les intérêts fondamentaux de la majorité du peuple» (《中国最广大人民的根本利益》). Sur la 《société de petit confort », voir Deng Xiaoping 邓小平, Deng Xiaoping wenxuan di'erjuan 邓小平文选第二卷 (Écrits choisis de Deng Xiaoping, Volume 2), Pékin 北京, Renmin chubanshe 人民出版社, 2002, p.237-238). La xiaokang shehui 小康社会 est un objectif politique déterminé par Deng Xiaoping dans le cadre de sa politique de "modernisation" de la Chine (xiandaihua 现代化) élaborée dès 1979. La finalité de cette politique était l'édification et la concrétisation en Chine d'une société où chacun des individus vivrait dans une situation d'aisance matérielle suffisante pour être à l'abri des besoins essentiels.

17 Ce qui distingue Hu Jintao des autres dirigeants qui l'ont précédé, c'est qu'il aura finalement accédé à la reconnaissance de ses pairs assez rapidement, en l'espace de trois ans. Jiang Zemin, lui, a apporté ses «trois représentations » en 2001 seulement, soit douze ans après son accession au poste de secrétaire général, et un an seulement avant la passation de pouvoir à $\mathrm{Hu}$ Jintao. Dans la rhétorique du Parti, il est toujours indispensable de souligner et de marquer la continuité idéologique entre les différents dirigeants. Ainsi, dans les allocutions officielles, Hu Jintao (ainsi que tous les hauts dirigeants chinois) soulignent systématiquement, telle une litanie, comment telle ou telle politique s'inscrit dans la continuité directe du «marxisme-léninisme, de la pensée de Mao Zedong, de la théorie de Deng Xiaoping et de l'importante pensée des "trois représentations" " (makesi liening zhuyi, maozedong sixiang, dengxiaoping lilun he sangedaibiao zhongyao sixiang 马克思列宁主义、毛泽东思想、邓小平理论和“三个代表” 重要思想). Ce lignage idéologique, essentiellement discursif, est fondamental : il permet de légitimer dans le discours (et donc en apparence) une décision politique quand bien même celle-ci serait dans les faits contraire à la pensée socialiste (par exemple, les «trois représentations », qui marquent l'éloignement définitif d'avec la base populaire du socialisme); plus important, il permet surtout de donner une cohérence, et donc une légitimité, à soixante années marquées idéologiquement et politiquement par un grand nombre de ruptures et de divergences. Le rappel incessant, non pas à l'histoire ou au passé, mais aux contributions idéologiques des dirigeants passés assoit l'immuabilité du Parti et légitime son exercice continu du pouvoir.

18 Voir par exemple Agnès Andresy, Who's Hu: le président chinois Hu Jintao, sa politique et ses réseaux, Paris, L'Harmattan, 2008, pp.161-162 ; John Delury, " "Harmonious" In China: The ancient sources of modern doctrine », Policy Review, $\mathrm{n}^{\circ} 148$, avril-mai 2008.

19 Cette "preuve" a visiblement échappé à tous les acteurs politiques et économiques des années 1990 qui, en toute hypocrisie, justifiaient la reprise d'échanges commerciaux avec la Chine malgré la répression de Tiananmen qu'ils dénonçaient vigoureusement quelques mois auparavant en avançant le principe, soi-disant infaillible, que la croissance économique allait nécessairement apporter la démocratie et développer les droits de l'homme en Chine. Vingt ans plus tard force est de reconnaître qu'ils avaient bien entendu tort. Soulignons néanmoins, d'un autre côté, qu'il n'est pas nécessairement absurde de parler d'un hégémonisme des "valeurs occidentales", dont la revendication est tout autant discutable. Le concept de démocratie a souvent été déformé et utilisé de manière abusive pour justifier des pratiques intrusives tout à fait intéressées (la seconde guerre irakienne de 2003 en est le parfait exemple). Quant aux droits de l'homme, pour "universels" qu'ils soient depuis 1948, nombre de pays occidentaux, les premiers à dénoncer, justement, les abus du gouvernement chinois, sont loin d'être irréprochables en la matière.

20 Pierre Gentelle, "L'instrumentalisation du nom de Confucius par la Chine populaire ", Monde chinois, ${ }^{\circ} 8$, été/automne 2006, note 6, p.53. Pierre Gentelle est géographe et travaille sur l'Asie orientale.

21 Bernard Cassen, «Du bon usage des "valeurs asiatiques" : un concept sur mesure né à Singapour et à Kuala-Lumpur », Le Monde Diplomatique, n4497, août 1995, p.2. Bernard 
Cassen est l'ancien directeur du Monde diplomatique.

22 Guo Baogang \& Guo Sujian, "China in Search of Harmonious Society », in Guo Suijian et Guo Baogang, China in Search of Harmonious Society, Lahham, Rowman \& Littlefield, 2008, p.2. Jiang Zemin avait mentionné l'édification d'une société harmonieuse comme l'un des six objectifs à accomplir dans le but d'atteindre la « société de petit confort sur tous les plans ».

23 Guo Baogang \& Guo Sujian, «China in Search of Harmonious Society », p.3 (« the classical trade-off between efficiency and equity », les termes entre crochets ont été rajoutés par nous).

24 Guo Baogang \& Guo Sujian, « China in Search of Harmonious Society », p.3 (« singleminded pursuit of economic growth and an achievement-based legitimacy (...) is being overshadowed by social and economic injustice, income and regional disparities, and environmental disasters », les termes entre crochets ont été rajoutés par nous).

25 Il y parviendra dans le discours, en inventant des concepts hybrides comme " l'économie socialiste de marché », mais pas dans la réalité.

26 Le terme « habits neufs » est utillisé en référence au travail de Simon Leys, Les Habits neufs du président Mao, Paris, Champ Libre, 1977. Pierre Ryckmans, alias Simon Leys, fut le premier à traiter la « Révolution culturelle » pour ce qu'elle était : une lutte de pouvoirs et pour le pouvoir, abusivement déguisée en apologie des forces créatives. L'auteur eut le mérite de publier cet ouvrage dans le climat pro-maïste de l'époque en France, très hostile à la critique. Sa carrière universitaire dut en pâtir par la suite

27 Guo Baogang \& Guo Sujian, "China in Search of Harmonious Society », p.4 (« are clearly not interested in building a modern utopia; instead, they are interested in the cultural reconstruction through political framing. Framing involves the packaging of new ideas in such a way as to make reference to an existing popular communication frame, thus to encourage certain interpretation and discourage others»).

28 Extrait de l'«Allocution du président Hu Jintao lors du séminaire organisé pour les hauts dirigeants et les cadres provinciaux autour de la thématique "augmenter notre capacité à construire une société harmonieuse socialiste” » du 19 février 2005.

$29 \mathrm{Li} J i$ 礼记 est la restauration d'une compilation de textes, qu'auraient collectés Confucius et rédigés ses disciples, traitant de l'organisation sociale sous la dynastie des Zhou 周 (environ 1050 - 256 av. J.-C.). Confucius (Kongzi 孔子 ou Kongfuzi 孔夫子) est un philosophe chinois qui vécut entre 551 et 479 av. J.-C. Il n'existe pas d'écrits qui puissent lui être proprement attribués, mais un ensemble de ses réflexions et de ses propos ont été recueillis et compilés par ses disciples, notamment dans les Entretiens (Lunyu 论语) Sa pensée a été interprétée pour former une idéologie d’État, le confucianisme, sous la dynastie Han 汉 (206 av.J.-C. - 220), basée sur le respect de la hiérarchie et de l'ordre établi, et la soumission via un ensemble de conventions sociales (li 礼). Sur Confucius, voir Confucius, Entretiens avec ses disciples, trad. du chinois par André Lévy, Paris, Flammarion, 1993 ; Pierre Ryckmans, Les Entretiens de Confucius, Paris, Gallimard, 1989.

30 Extrait de l'« Allocution du président Hu Jintao lors du séminaire organisé pour les hauts dirigeants et les cadres provinciaux autour de la thématique "augmenter notre capacité à construire une société harmonieuse socialiste" " du 19 février 2005. Les éléments entre crochets ont été rajoutés par nous.

31 Guo Baogang, «Utopias of Reconstruction : Chinese Utopianism Form Hong Xiuquan to Mao Zedong », Journal of Comparative Asian Development, vol. 2, $\mathrm{n}^{\circ} 2,2003$, pp.97-210). L'un des slogans majeurs de la campagne des Jeux Olympiques en Chine était précisément, « one world, one dream » (tongyige shijie, tongyige mengxiang 同一个世界, 同一个梦想). Ce slogan, qui signifiait l'idée d'une communauté mondiale retrouvée autour du dépassement de soi prôné par les Jeux Olympiques, signifiait également la volonté de la Chine de marquer son appartenance à la "communauté mondiale", en dépit des "spécificités" (guoqing 国情), celles de son régime politique totalitaire, qu’il allait falloir dorénavant accepter et dépasser au profit d'une grande communauté mondiale réconciliée. C'est dans ce sens qu'il faut comprendre le " monde harmonieux » (hexie shijie 和谐世界) que le PCC appelle à édifier, partie intégrante du discours de la « société harmonieuse » et qui constitue la ligne politique officielle de la République populaire de Chine dans ses relations internationales. C'est au nom de ce concept, un " monde harmonieux ", un monde unifié, un, que la Chine ne consent pas de se "mêler" des affaires intérieures des pays africains chez qui elle se fournit en pétrole, et à qui elle vend ses services et ses armes (l'exemple du Soudan est assez connu et reconnu). Ce "monde harmonieux » prôné par Hu Jintao est un monde où les conflits et les injustices n'existent 
plus, parce qu'ils se taisent et ne peuvent s'exprimer. Le concept de «société harmonieuse » renferme exactement les mêmes mécanismes sémantiques et idéologiques.

32 Le confucianisme est une réinterprétation idéologique de la pensée développée par Confucius.

33 Shi Zhongwen, «Traditional Culture Embodied in Confucianism », in Guo Suijian et Guo Baogang, China in Search of Harmonious Society, Lahham, Rowman \& Littlefield, 2008, p.37. Shi Zhongwen est professeur de littérature comparée.

34 Wolfgang Bauer, China and the Search for Happiness, trad. de l'allemand par Michael Shaw, New York, Seabury, 1976, p.21 (« equality rests on the consistency of the system of government in its totality and as it affects everyone »), p.37 (" life as decreed by fate. Together with his name, it is imposed on man by the gods (...) a mission heaven assigns to man as it gives him life, and to which he had to adapt as closely as possible by conscientiously fulfilling his part in human society »). Notre méconnaissance de la langue allemande nous a obligé à nous reporter sur la traduction anglaise de l'ouvrage de Wolfgang Bauer.

35 Jean-François Billeter, Contre François Jullien, Paris, Allia, 2006, pp.26-27 (les caractères chinois ont été rajoutés par nous).

36 Georges Orwell a parfaitement démontré, dans le trop sous-estimé Animal Farm (La Ferme des Animaux), comment s'installe le totalitarisme au sein d'une collectivité d'individus : la répartition "naturelle" des tâches, quelle que soit sa motivation, crée les conditions de formation d'une élite auto-proclamée (la division sociale s'installe de manière imperceptible dans le récit quand les cochons expliquent qu'ils ne sont naturellement doués en rien, mais qu'ils savent réfléchir : ils s'installent de ce fait dans la supervision des tâches de la nouvelle république des animaux que tous viennent de créer), et le contrôle de l'information, qui permet d'écrire (et de réécrire) l'histoire en permanence, entrave la transmission de la mémoire, et in fine la fin de l'histoire (voir George Orwell, Animal Farm, Londres, Longman, 1996).

37 Bauer, China and the Search for Happiness, p.22 («the fundamental equation of an ideal world order, calm and equality can thus already be found in Confucius, although its form rather reminds us a uniform stabilization of inequality »).

38 Billeter, Contre François Jullien, p.18.

39 Billeter, Contre François Jullien, pp.18-19.

40 Car le confucianisme n'est pas autre chose qu'une idéologie de la domination. Sur le confucianisme à l'époque moderne et contemporaine, voir Flora Blanchon \& Rang-Ri Park-Barjot (Dir.), Le Nouvel âge de Confucius : Modern Confucianism in China and South Korea, Paris, PUPS, 2007 ; Wei Zhengtong 韦政通, Rujia yu xiandai Zhongguo 儒 家与现代中国 (Confucianisme et Chine moderne), Taipei 台北, Dongda tushu 东大图书, 2001.

41 Billeter, Contre François Jullien, pp.28.

42 Anne-Marie Broudehoux, "Civilizing Beijing: Social beautification, civility, and citizenship at the 2008 Olympics », in Graeme Hayes \& John Karamichas, Mega-events and Civil Societies: Environment and Globalisation, Accommodation and Resistance, London, Palgrave-Macmillan, 2010 (à paraître), p.6 (du manuscrit). Nous tenons à remercier Anne-Marie Broudehoux de nous avoir permis d'utiliser ce travail encore non publié.

43 Leïla Choukroune \& Antoine Garapon, « Les Normes de l'harmonie chinoise : un droit disciplinaire comme stabilisateur social », Perspectives Chinoises, $\mathrm{n}^{\circ}$ 2007/3, p.51.

44 L'époque où Confucius était honni au même titre que le « traitre Lin Biao » (lors de la campagne fanlin fankong 反林反孔 de la 《Révolution culturelle») est bien loin. Confucius, au même titre que Cervantès pour l'Espagne, est le digne représentant de la Chine dans le monde via les Instituts Confucius. Sur l'instrumentalisation des concepts traditionnels par le PCC en quête de légitimité, voir Werner Meissmer, "Réflexions sur la quête d'une identité nationale et culturelle en Chine ", Perspectives chinoises, $\mathrm{n}^{\circ} 97$, septembre-décembre 2006, pp.45-59.

45 Sébastien Billioud, " "Confucianisme", "tradition culturelle" et discours officiels dans la Chine des années 2000 ", Perspectives Chinoises, n²007/3, pp.53-54. Sébastien Billioud y voit une "redécouverte populaire » de la pensée confucéenne. Mais dans une société du spectacle, où les rapports de pouvoir et de domination s'exercent en majeure partie via les média, il n'y a pas de « redécouverte populaire », il n’y a essentiellement que propagande et matraquage publicitaire. Billioud le reconnaît plus loin. « La fièvre pour les 
études nationales (c'est-à-dire les études classiques) que l'on observe alors, sans pouvoir être réduite à une stratégie politique, n'est pas pour autant sans lien avec l'incitation des autorités » (Billioud, « "Confucianisme", "tradition culturelle" et discours officiels dans la Chine des années 2000 », p.56). Sur l'engouement autour des études nationales (guoxue 国学), Chen Yan établira le même constat : cette " fièvre » est en (grande) partie soutenue et alimentée par les autorités (voir Chen Yan, L'Eveil de la Chine : les bouleversements intellectuels après Mao (1976-2002), La Tour d'Aigues, Aube, 2002, p.304, note 286). Sébastien Billioud. C'est essentiellement une quête identitaire doublée d'une quête communautaire dans un contexte de déchirure des tissus sociaux (nous n'insisterons jamais assez sur les conséquences des expulsions dans les grandes villes en terme de destruction des mémoires (individuelles et urbaines) et de déconstruction des affinités de quartiers, une fois que les différentes existences individuelles se retrouvent éparpillées en périphéries ou en banlieues) qui ont poussé un grand nombre de Chinois à se tourner vers la secte Falungong (falun dafa 法轮大法). Si nous insistons sur le fait que ce "mouvement spirituel" est une véritable secte (avec gourou, mystifications, croyances mystiques, extorsions de fonds, et manipulations des esprits) poursuivant un certain agenda politique, soulignons également qu'une grande majorité de ses membres n'étaient pas nécessairement crédules pour autant. Sur la secte Falungong, voir Maria Hsia Chang, Falungong, secte chinoise: un défi au pouvoir, trad. de l'américain par Geneviève Brzustowski, Paris, Autrement, 2004; nous regrettons néanmoins l'absence d'une approche anthropologique et d'une étude sur les motivations des individus.

46 Pierre Bourdieu, Langage et pouvoir symbolique, Paris, Seuil, 2001, p.210. Sur le mouvement pro-démocratique de Tiananmen, voir par exemple Gregory B. Lee, China's Lost Decade, Lyon, Tigre de Papier, 2009 ; Jean-Philippe Béja, Michel Bonnin \& Alain Peyraube, Le Tremblement de terre de Pékin, Paris, Gallimard, 1991; Han Minzhu, Cries for Democracy: Writing and Speeches from the 1989 Chinese Democracy Movement, Princeton, Princeton University Press, 1990.

47 Bourdieu, Langage et pouvoir symbolique, p.210. Nous n'insisterons pas plus sur cette question de l'impact du discours de la «société harmonieuse » sur les consciences (un point sur lequel il nous est raisonnablement impossible de nous prononcer), mais nous nous contenterons de souligner que le discours de la " société harmonieuse ", parce qu'il s'inscrit dans un contexte et un climat favorables à sa présence, peut être convaincant sans pour autant arriver à convaincre : c'est-à-dire qu'il peut très bien développer un ensemble d'idées raisonnables et pleines de bon sens, sans pour autant être cru.

48 Nous insistons sur ce point : la présence massive (quantitative) des expressions du PCC dans l'espace public chinois est avérée et manifeste ; son emprise (qualitative) sur les consciences l'est beaucoup moins. D'une manière générale, en Chine, nous avons régulièrement constaté, autant chez les membres du Parti que chez le simple quidam, un recul manifeste, pour ne pas dire un réel détachement, d'avec cette propagande : ce n'est pas nécessairement le message qui est négligé ou ignoré, mais c'est l'émetteur du message, le Parti, qui n'est plus nécessairement cru (ou qui ne croit même plus à ses propres professions de foi). Mais nous ne discuterons pas ici de l'impact des messages de propagande sur les consciences, pour deux raisons: cette évaluation nous semble impossible à réaliser (et c'est d'ailleurs là, à nos yeux, la grande limite de la sociologie actuelle pratiquée en Chine, cette incapacité à reconnaître l'ineptie des enquêtes quantitatives (d'opinion ou autres), en ce sens qu'elles ne peuvent rien enseigner, hormis qu'il est impossible d'en exploiter les "résultats" tant les filtres et les précautions dans l'expression et la prise de parole des individus en Chine sont nombreux); ce n'est pas là le propos de notre travail, qui se veut être une étude des messages en soi, de cet espace "interstitiel” entre les émetteurs et les récepteurs.

49 Son impact en terme de présence quantitative s'est retrouvé dilué dans un champ maintenant également parsemé d'autres signes (publicités marchandes) qui rendent l'espace public visuellement "cacophonique".

50 Il est utile de souligner que le terme chinois xuanchuan 宣传 (propagande) est, contrairement au français, un terme neutre qui n'est chargé d'aucune connotation péjorative.

51 Luc Boltanski \& Pierre Bourdieu, La Production de l'idéologie dominante, Paris, Demopolis, 2008, p.120. Toute cette circulation de textes, d'expressions et d'opinions "para-officielles" (en ce sens qu'elles ne proviennent pas d'agents de l'Etat mais de personnalités "civiles") constituent une grande partie du corpus idéologique de la « société harmonieuse » et sont régulièrement publiés et diffusés par les deux canaux officiels (pour ne pas dire organiques) du Parti, à savoir l'agence de presse Chine nouvelle (Xinhuashe 新 华社) et le Quotidien du peuple (Renmin ribao 人民日报).

52 Thierry Guilbert, Le Discours idéologique ou la Force de l'évidence, Paris, 
L'Harmattan, 2008, p.86.

53 《在人均国内生产总值突破1000美元之后, 经济社会发展就进入了一个关键阶段》; 《既 有因为举措得当从而促进经济快速发展和社会平稳进步的成功经验”, extrait de l'« Allocution du président Hu Jintao lors du séminaire organisé pour les hauts dirigeants et les cadres provinciaux autour de la thématique "augmenter notre capacité à construire une société harmonieuse socialiste” » du 19 février 2005.

54 《维护好、发展好最广大人民的根本利益》; 《必须加强社会建设和管理, 营造良好的人 际环境, 保持良好的社会秩序, 维护社会稳定, 保证广大人民群众安居乐业》, extrait de l'« Allocution du président Hu Jintao lors du séminaire organisé pour les hauts dirigeants et les cadres provinciaux autour de la thématique "augmenter notre capacité à construire une société harmonieuse socialiste” » du 19 février 2005.

55 Extrait de l'« Allocution du président Hu Jintao lors du séminaire organisé pour les hauts dirigeants et les cadres provinciaux autour de la thématique "augmenter notre capacité à construire une société harmonieuse socialiste" " du 19 février 2005. La "société harmonieuse» se veut également être la nouvelle politique de la Chine en matière d'affaires étrangères et de diplomatie. Une partie de cette allocution évoque d'ailleurs la construction d'un monde harmonieux. Cet aspect "international" de la société harmonieuse est important, et le gouvernement chinois en fait notamment usage dans ses relations avec les pays africains notamment, le Soudan en particulier, pour justifier sa collaboration économique avec des régimes politiques contestés pour leur autoritarisme, en arguant que seul le respect des différences permettra l'harmonie et la paix mondiales. Au sujet des relations entre la Chine et l'Afrique, voir Chris Alden \& Christopher R. Hughes, "Harmony and Discord in China's Africa Strategy: Some Implications for Foreign Policy », China Quaterly, vol.199, septembre 2009, pp.563-584 ; Julia C. Strauss, " The Past in the Present: Historical and Rhetorical Lineages in China's Relations with Africa », China Quaterly, vol.199, septembre 2009, pp.777-795 ; voir également Michel Beuret, Serge Michel \& Paolo Woods, La Chinafrique, Paris, Grasset, 2008.

56 Pierre Bourdieu, Contre-feux 2, Paris, Raisons d'agir, 2001, p.10.

57 Jean-Marc Vernier, « Modernisation », in Pascal Durand (Dir.), Les nouveaux mots du pouvoir : abécédaire critique, Bruxelles, Aden, 2007, p.312. Dans cette définition, Jean-Marc Vernier parlait spécifiquement de la France, mais cette approche est, nous semble-t-il, parfaitement applicable à tout autre pays régi par les règles de la mondialisation et du capitalisme libéral.

58 《中国共产党的领导和我国的社会主义制度, 为构建社会主义和谐社会提供了最根本的 保证》, extrait de l'《 Allocution du président Hu Jintao lors du séminaire organisé pour les hauts dirigeants et les cadres provinciaux autour de la thématique "augmenter notre capacité à construire une société harmonieuse socialiste” » du 19 février 2005.

59 《经过新中国成立以来特别是改革开放以来的不断发展》; 《在我国, 各阶层、各党派、 各民族、各团体政治上享有平等地位》, extrait de l'《 Allocution du président Hu Jintao lors du séminaire organisé pour les hauts dirigeants et les cadres provinciaux autour de la thématique "augmenter notre capacité à construire une société harmonieuse socialiste" » du 19 février 2005.

60 《我国仍然处于并将长期处于社会主义初级阶段》; 《建成社会 主义和谐社会任重道远。 同建设社会主义现代化国家要经历一个很长历史过程一样, 构建社会主义和谐社会也是一 个需要随着经济、政治、文化的发展而不断推进的很长历史过程》, extrait de l'《Allocution du président Hu Jintao lors du séminaire organisé pour les hauts dirigeants et les cadres provinciaux autour de la thématique "augmenter notre capacité à construire une société harmonieuse socialiste” » du 19 février 2005.

61 Les objectifs de la «société de petit confort » prévus depuis le début des années 1980 pour l'an 2000 n'ont par exemple jamais été atteints. Un certain nombre de critères auraient dû être satisfaits, tels que : la consommation de marchandises ne doit plus se réduire aux besoins vitaux mais être également axée sur le plaisir et le développement personnel; les individus seront propriétaires de leur logement et seront capables de voyager au moins une fois par an ; le fossé entre les revenus urbains et ruraux se réduira ; l'espérance de vie moyenne atteindra les 72 ans, etc. L'échec n'a jamais été reconnu, et a été au contraire retourné par une subtilité linguistique : dans sa rhétorique officielle, le Parti affirme que la Chine est depuis 2000 une "société de petit confort », soulignant juste que ce confort n'est pas présent partout et « sur tous les plans » (bu quanmian de 不 全面的).

62 Extrait de l'" Allocution du président Hu Jintao lors du séminaire organisé pour les hauts dirigeants et les cadres provinciaux autour de la thématique "augmenter notre capacité à construire une société harmonieuse socialiste” » du 19 février 2005. 
63 《始终把最广大人民的根本利益作为党和国家工作的根本出发点和落脚点》, extrait de l'« Allocution du président Hu Jintao lors du séminaire organisé pour les hauts dirigeants et les cadres provinciaux autour de la thématique "augmenter notre capacité à construire une société harmonieuse socialiste” » du 19 février 2005.

64 Ce sont les consommateurs moyens urbains, une « classe moyenne » créée par le Parti, constituée en grande partie de fonctionnaires pour qui l'accès à la propriété immobilière a été considérablement facilité par l'État dans les années 1990 (par le biais notamment de prêts bancaires très attractifs). En offrant à certains l'accès à la propriété, le Parti-État se ménage une « classe » d'individus nécessairement favorable à l'ordre social établi (et donc à sa pérennité) puisque celui-ci leur est, en définitive, très favorable.

65 Extrait de l'«Allocution du président Hu Jintao lors du séminaire organisé pour les hauts dirigeants et les cadres provinciaux autour de la thématique "augmenter notre capacité à construire une société harmonieuse socialiste” » du 19 février 2005.

66 Jean-François Billeter, Chine trois fois muette, Paris, Allia, 2006, p.61.

67 Extrait de l'" Allocution du président $\mathrm{Hu}$ Jintao lors du séminaire organisé pour les hauts dirigeants et les cadres provinciaux autour de la thématique "augmenter notre capacité à construire une société harmonieuse socialiste” » du 19 février 2005.

68 《切实保持经济持续快速协调健康发展》; 《解决我国 (...)面临的许多矛盾和问题(...), 关 键还是要靠发展》, extrait de l'《Allocution du président Hu Jintao lors du séminaire organisé pour les hauts dirigeants et les cadres provinciaux autour de la thématique "augmenter notre capacité à construire une société harmonieuse socialiste" » du 19 février 2005 .

69 《才能创造更多就业机会, 才能不断满足人民群众多方面的需求。经济发展(...)要协调健 康, 这是人民群众不断提高生活水平的重要保证》, extrait de l'《Allocution du président $\mathrm{Hu}$ Jintao lors du séminaire organisé pour les hauts dirigeants et les cadres provinciaux autour de la thématique "augmenter notre capacité à construire une société harmonieuse socialiste” » du 19 février 2005.

70 《广大农民日子过好了、素质提高了, 广大农村形成安定祥和的局面了》, extrait de l'«Allocution du président Hu Jintao lors du séminaire organisé pour les hauts dirigeants et les cadres provinciaux autour de la thématique "augmenter notre capacité à construire une société harmonieuse socialiste” » du 19 février 2005.

71 Extrait de l'«Allocution du président Hu Jintao lors du séminaire organisé pour les hauts dirigeants et les cadres provinciaux autour de la thématique "augmenter notre capacité à construire une société harmonieuse socialiste" » du 19 février 2005.

72 《一个社会是否和谐, 一个国家能否实现长治久安, 很大程度上取决于全体社会成员的 思想道德素质》, extrait de l' «Allocution du président Hu Jintao lors du séminaire organisé pour les hauts dirigeants et les cadres provinciaux autour de la thématique "augmenter notre capacité à construire une société harmonieuse socialiste” 》 du 19 février 2005.

73 《引导全体人民坚定中国特色社会主义信念》; 《以爱国主义为核心的民族精神》; 《改革创 新为核心的时代精神》, extrait de l'《Allocution du président Hu Jintao lors du séminaire organisé pour les hauts dirigeants et les cadres provinciaux autour de la thématique "augmenter notre capacité à construire une société harmonieuse socialiste" » du 19 février 2005. Insister sur «l'esprit de notre temps » est une stratégie discursive qui enferme l'interlocuteur dans une dualité modernisme contre passéisme qui empêche l'exercice de l'esprit critique. Dans ce discours, s'opposer aux réformes en Chine équivaut à s'opposer à "l'esprit de l'époque", et donc, nécessairement, à faire preuve d'un "passéisme réactionnaire" voir d'une "pensée féodale" (fengjian sixiang 封建思想), un lexique régulièrement avancé en opposition à la modernité (xiandai 现代).

74 Extrait de l'« Allocution du président Hu Jintao lors du séminaire organisé pour les hauts dirigeants et les cadres provinciaux autour de la thématique "augmenter notre capacité à construire une société harmonieuse socialiste” » du 19 février 2005. On trouve les prémisses d'une moralisation de la question sociale dès 2001, avec la mise en place du « Programme de mise en œuvre de la construction d'une morale citoyenne » (gongmin daode jianshe shishi gangyao 公民道德建设实施纲要). Approuvé par le quinzième Comité central du Parti communiste chinois le 20 septembre 2001, ce programme vise à éduquer la population chinoise à la citoyenneté, une citoyenneté envisagée essentiellement dans sa dimension morale et résumée dans la mise en place de "bonnes" mœurs et de "bonnes" pratiques, susceptibles de créer un bon « climat social » (shehui fenwei 社会氛围). Sur ce programme, voir Xu Qixian 许启贤, Wenming beijingren «gongmin daode jianshe shishi gangyao 》shimin duben 文明北京人 《公民道德建设实施纲要》市民读本 (programme de mise en œuvre de la construction d'une morale citoyenne du Pékinois civilisé, manuel du citadin), Pékin 北京, Beijing shifan daxue chubanshe 北京师范大学出版社, 2003. 
75 Extrait de l'« Allocution du président Hu Jintao lors du séminaire organisé pour les hauts dirigeants et les cadres provinciaux autour de la thématique "augmenter notre capacité à construire une société harmonieuse socialiste” » du 19 février 2005.

76 Limore Yagil, «L'Homme nouveau » et la Révolution nationale de Vichy (1940-1944), Villeneuve-d'Ascq, Septentrion, 1997, p.11. La création d'un « homme nouveau » a d'abord été l'une des finalités des utopies révolutionnaires avant de devenir le credo des régimes totalitaires. Ce fut par exemple le cas sous le régime maoïste lors de la « révolution culturelle » (wenhua dageming 文化大革命), mais bien avant déjà avec la création de la «Chine nouvelle » (xin Zhongguo 新中国).

77 Que la question de la moralité vise en particulier la population chinoise paupérisée est un discours bien relayé en Chine. Citons à titre d'exemple l'ouvrage de l'économiste Mao Yushi 茅于轼, Perspectives pour la moralité des Chinois (Zhongguoren de daode qianjing 中国人的道德前景), dans lequel un chapitre entier, intitulé " reconstruire le Chinois » (《再造中国人»), décline le discours de la “crise de la moralité" des Chinois et souligne que 《la morale est un facteur de stabilité pour une société moderne 》 (《道德是现代社会的稳 定剂》) (voir Mao Yushi 茅于轼, Zhongguoren de daode qianjing 中国人的道德前景 (Perspectives pour la moralité des Chinois), Canton 广州, Jinan daxue chubanshe 暨南大 学出版社, 2003, p.177). La première page de l'ouvrage, la page de titre, est sans équivoque : surplombé d'une dédicace par laquelle l'auteur " dédie ce livre à tous ceux qui se sentent concernés par le développement de la société chinoise 》 (《谨以此书献给所有关 心中国社会发展的人们 »), traîne en dessous du titre une photo en noir et blanc d'une file de mingong 民工 avec sacs et baluchons. L'amalgame est donc en place: le développement de la société chinoise passe par l'éducation morale de ses pauvres et ses mingong.

78 Michel Foucault, L’Ordre du discours, Paris, Gallimard, 1971, p.46.

79 Guilbert, Le Discours idéologique ou la Force de l'évidence, p.52.

80 Précisons, encore une fois, que nous ne nous intéressons pas à la réception de ce discours au sein de la population chinoise. Nous ne disons donc pas que la «société harmonieuse " convainc, ni qu'elle exerce une pression effective sur les conceptions morales des individus, et encore moins qu'elle parvient inévitablement à transformer la perception qu'ont les individus du monde. Nous disons seulement qu'elle y prétend. Pour résumer notre propos, nous ne disons pas que la société harmonieuse parvient à transformer l'ensemble de la population chinoise en homo harmonicus, nous disons simplement que ce discours est présent, et que l'on peut y distinguer des tendances et des motivations. Par ailleurs, un discours peut être totalisant, c'est-à-dire s'adresser à la totalité de la société prise sous tous ses aspects, sans nécessairement être dominant, c'està-dire imprégner effectivement et durablement les consciences.

81 Le texte de l'allocution de Hu Jintao du 4 mars 2006, intitulée « Mettons solidement en place la conception socialiste de l'honneur et de la honte » (« Laogu shuli shehuizhuyi rongruguan 牢固树立社会主义荣辱观》) est paru dans le Renmin ribao quelques semaines plus tard (Hu Jintao 胡锦涛, “ Laogu shuli shehuizhuyi rongruguan 牢固树立社会主义荣 辱观 ", Renmin ribao 人民日报, édition du 28 avril 2006). Ce texte est par ailleurs consultable sur le site Internet de l'agence Chine nouvelle (xinhuashe 新华社), voir $\mathrm{Hu}$ Jintao 胡锦涛, “ Laogu shuli shehuizhuyi rongruguan 牢固树立社会主义荣辱观 》, Xinhuawang 新华网, publié le 27 avril 2006, consulté le 12 août 2009 sur http://news.xinhuanet.com/newscenter/2006-04/27/content_4482066.htm. La Conférence consultative politique du peuple chinois (zhongguo renmin zhengzhi xieshang huiyi 中国人民政治协商会议) est une assemblée sans pouvoir décisionnaire, regroupant l'ensemble des huit autres partis politiques démocratiques chinois "autorisés", gage du centralisme démocratique affirmé par le PCC.

82 Une véritable campagne idéologique a pris le relais de cette allocution, et les « Huit honneurs et huit hontes » se sont retrouvés affichés durant de nombreux mois dans les lieux publics (ils le sont encore dans certains endroits en 2009). Le ministre de l'éducation Zhou Ji 周济, très enthousiaste, déclara même à l'époque «vouloir faire entrer la conception socialiste de l'honneur et de la honte dans les salles de classe, et dans la tête de chaque étudiant » (《要把社会主义荣辱观(...)引入课堂、引入学生头脑》) (Beijing chenbao 北京晨报, 《Jiaoyubu buzhang zhouji: “barong bachi” yao yinru ketang 教育部部长周济: “八荣八耻”要引入课堂”, Beijing chenbao 北京晨报, édition du 14 mars 2006).

83 《全面建设小康社会、加快推进社会主义现代化, 要求我们必须把发展社会主义先进文 化放到十分突出的位置》, extrait de l'allocution de Hu Jintao "Mettons solidement en place la conception socialiste de l'honneur et de la honte » du 4 mars 2006.

84 Le terme wenhua 文化 se traduit également parfois par « civilisation », à comprendre comme désignant l'ensemble des pratiques culturelles d'une communauté. La wenhua 文 
化 (culture) fait d'ailleurs l'objet d'un programme de développement gouvernemental. Le dernier en date, le "Programme de développement de la culture inscrit dans le onzième plan quinquennal » (guojia “shiyiwu” shiqi wenhua fazhan guihua gangyao 国家“十一五” 时期文化发展规划纲要), date de septembre 2006, et insiste particulièrement sur la "qualité morale des citoyens" et sur l'importance de la " conception socialiste de l'honneur et de la honte » comme bases de la culture socialiste (Renmin chubanshe 人民出版社, Guojia “shiyiwu” shiqi wenhua fazhan guihua gangyao 国家“十一五”时期文化发展规划纲 要 (Programme de développement de la culture inscrit dans le onzième plan quinquennal), Pékin 北京, renmin chubanshe 人民出版社, 2006) . Comme l'explique Sébastien Billioud, la culture, selon ce programme, "doit fournir les "ressources spirituelles" pour construire une société d'harmonie socialiste et parvenir à une "société de bien-être moyen” (xiaokang shehui 小康社会)" (Billioud, “Confucianisme", "tradition culturelle" et discours officiels dans la Chine des années 2000 », pp.58-59). Le texte intégral du "Programme de développement de la culture inscrit dans le $\mathrm{XI}^{\circ}$ plan quinquennal » est consultable sur le site du Quotidien du peuple (Renmin ribao 人民日报, “Guojia “shiyiwu” shiqi wenhua fazhan guihua gangyao (quanwen) 国家“十一五”时期文化 发展规划纲要(全文)», Renminwang 人民网, publié le 13 septembre 2006, consulté le 17 octobre 2008 sur http://culture.people.com.cn/GB/22226/71018/4814170.html).

85 《发展教育科学文化, 培育有理想、有道德、有文化、有纪律的社会主义公民》, extrait de l'allocution de Hu Jintao "Mettons solidement en place la conception socialiste de l'honneur et de la honte » du 4 mars 2006.

86 《当今世界的综合国力竞争, 说到底是民族素质竞争》, extrait de de l'allocution de Hu Jintao " Mettons solidement en place la conception socialiste de l'honneur et de la honte » du 4 mars 2006. Le terme suzhi 素质 (qualité) est régulièrement utilisé dans le discours dominant pour juger et déprécier, c'est-à-dire utilisé à la forme négative sur des individus considérés comme manquant de suzhi. Ce discours vise particulièrement la population rurale. Sur le terme suzhi 素质, voir Ann Anagnost, «The Corporeal Politics of Quality (Suzhi) », Public Culture, vol.16, $\mathrm{n}^{\circ} 2,2004$, p.195. Sur la notion de suzhi 素质, voir également Andrew Kipnis, "Suzhi: a Keyword Approach », The China Quaterly, n ${ }^{\circ} 86$, 2006, pp.295-313.

87 Loïc Wacquant, Punir les pauvres : le nouveau gouvernement de l'insécurité sociale, Marseille, Agone, 2004, pp.26-27.

88 Mingong 民工 est la contraction de nongmin 农民 (paysan) et de gongren 工人 (travailleur). Le terme désigne ces migrants venus des régions rurales chercher en ville une activité rémunérée. On estime à environ 150 millions leur nombre dans les grandes métropoles chinoises. Sans protection sociale (ou très peu), ils sont généralement l'objet de discrimination, assimilés à des « étrangers » (waidiren 外地人), et travaillent dans des secteurs d'activité difficiles et peu qualifiés (les travaux publics et l'immobilier entre autres). Sur les mingong, voir Eric Florence, «Les Débats autour des représentations des migrants ruraux », Perspectives Chinoises, $n^{\circ} 94$, mars-avril 2006, pp.13-26; Hein Mallee, " Migration, hukou and resistance », in Elizabeth J. Perry \& Mark Selden, Chinese Society: Change, Conflict and Resistance (2nd Edition), London \& New York, Routledge, 2003. Les xiagang 下岗 (dans sa forme complète : xiagang zhigong 下岗职工) sont des travailleurs du secteur public littéralement "descendus de leur poste”, “démobilisés”, c'està-dire sans affectation, sans poste. Ce sont dans les faits des personnes sans emploi, sans travail, mais dont l'ancien statut d'employé d'entreprise étatique ne permet pas le licenciement.

89 Extrait de l'allocution de $\mathrm{Hu}$ Jintao « Mettons solidement en place la conception socialiste de l'honneur et de la honte » du 4 mars 2006.

90 Extrait de l'allocution de $\mathrm{Hu}$ Jintao «Mettons solidement en place la conception socialiste de l'honneur et de la honte » du 4 mars 2006.

91 Par exemple, comme toutes les grandes entreprises chinoises, la compagnie chinoise Lenovo (anciennement Legend) est inévitablement «talentueuse » d'avoir racheté la branche PC du géant de l'informatique IBM en décembre 2004. A contrario, l'écrivain chinois naturalisé français Gao Xingjian 高行健, "banni” de Chine en 1987 pour le caractère anti-conformiste de ses livres, prix Nobel de littérature en 2000, n'est lui absolument pas «talentueux » parce que son œuvre n'est pas conforme à la «culture socialiste avancée ».

92 《要树立良好的社会风气», extrait de l'allocution de Hu Jintao 《 Mettons solidement en place la conception socialiste de l'honneur et de la honte » du 4 mars 2006.

93 Extrait de l'allocution de $\mathrm{Hu}$ Jintao «Mettons solidement en place la conception socialiste de l'honneur et de la honte » du 4 mars 2006. 
94 《要教育广大干部群众特别是广大青少年树立社会主义荣辱观》, extrait de l'allocution de Hu Jintao « Mettons solidement en place la conception socialiste de l'honneur et de la honte » du 4 mars 2006.

95 Extrait de l'allocution de $\mathrm{Hu}$ Jintao «Mettons solidement en place la conception socialiste de l'honneur et de la honte » du 4 mars 2006.

96 Sur le mythe de la concurrence loyale et parfaite, voir Bernard Maris, Antimanuel d'économie, Vol.1 les fourmis, Rosny, Bréal, 2003, pp.108-144.

97 Jean-Louis Rocca, La Condition chinoise : la mise au travail capitaliste à l'âge des réformes (1978-2004), Paris, Karthala, 2006, p.57.

98 Guy Debord, « Avertissement pour la troisième édition française », p.XII.

99 Michel Foucault, Surveiller et Punir : naissance de la prison, Paris, Gallimard, 1975, p.213

100 Cette possibilité de normaliser des situations de précarité par le biais des « Huit honneurs et huit hontes " n'a pas échappé aux employeurs. Le réalisateur Jean-Yves Cauchard dans son documentaire Made in China consacré aux travailleurs migrants (et par ailleurs très précieux dans son approche puisqu'il fait partie des rares productions à aborder la question du déracinement et de l'exil chez les mingong), montre le directeur d'une usine de taille moyenne consacrée à la production de marchandises destinées à l'exportation, s'adressant, accompagné du délégué du PCC de l'entreprise, à ses employés, en majorité mingong, lors d'une session d'étude idéologique (ces sessions de travail idéologique sont le relais au sein des entreprises des campagnes idéologiques initiées par le Parti). La session du jour porte sur les « Huit honneurs et huit hontes », et le patron lit à haute voix, un par un, chacun des huit préceptes devant une foule d'ouvriers plutôt apathiques et désintéressés. L’intérêt de la séquence, c'est que le directeur de l'usine interprète les «Huit honneurs et huit hontes » de manière à justifier les conditions de travail de ses ouvriers, et finalement révèle la véritable nature de cette campagne d'éducation morale en exhortant les employés « aimer le travail » car l'usine est « comme une grande famille» tout en précisant qu'il faut "servir le peuple », rajoutant que " chaque travailleur le sait bien, tout ce que nous faisons c'est pour servir le peuple » (voir Jean-Yves Cauchard, Made in China, Hikari productions, 2006 ; la séquence se situe à une quarantaine de minutes du début du film). Les " Huit honneurs et huit hontes », comme toutes les stratégies discursives mises en œuvre dans le cadre de la "société harmonieuse », s'avèrent être un instrument discursif destiné à discipliner les populations pauvres et à justifier et valoriser leur exploitation.

101 Les « Huit honneurs et huit hontes » présentent finalement un grand nombre de similitudes avec le «Travail, famille, patrie » prôné par le régime de Vichy, régime qui également visait à la création d'un "homme nouveau » (notons tout de même que les régimes politiques et les circonstances historiques sont, eux, tout à fait différents). A ce sujet, Jean-Marie Guillon, «La nature du régime de Vichy : sa philosophie politique », in Jean-Pierre Azéma, François Bédarida (Dir.), Vichy et les Français, Paris, Fayard, 1992, pp.167-188.

102 Précisons encore une fois que l'écart est grand entre les prétentions d'un discours officiel et sa réception, son impact réel sur les individus. Voir l'article de Edward Cody, " Eight-Step Program for What Ails China », The Washington Post, édition du 23 mars 2006.

\section{Pour citer cet article}

Référence électronique

Thomas BOUTONNET, « Traitement moral de la question sociale dans la « société harmonieuse » de Hu Jintao », Transtext(e)s Transcultures 跨文本跨文化 [En ligne] , 5 | 2009 , mis en ligne le 01 juin 2009, consulté le 10 mai 2011. URL :

http://transtexts.revues.org/271

\section{Auteur}

\section{Thomas BOUTONNET}

Thomas BOUTONNET est docteur en études chinoises et transculturelles, et a été directeur de l'Institut Confucius de Lyon (2009-2010). Chercheur au sein de l'Institut 
d'Études Transtextuelles et Transculturelles (www.iett.eu), il enseigne actuellement la langue et la société contemporaine chinoises à l'université de Lyon - Jean Moulin. Sa thèse, intitulée "Vers une "société harmonieuse" de consommation ? Discours et spectacle de l'harmonie sociale dans la construction d'une Chine "civilisée" (1978-2008) », aborde le processus de civilisation des populations paupérisées durant le passage à une économie de marché en Chine. Son travail de recherche actuel porte sur la déconstruction des discours idéologiques en Chine contemporaine, sur l'étude des mots du pouvoir chinois et sur une analyse approfondie du concept de civilisation. Thomas BOUTONNET has a PhD in Chinese and Transcultural Studies and was the executive director of the Lyon Confucius Institute in 2009-2010. He currently lectures in the Chinese department of the University of Lyon - Jean Moulin and is an active member of the Institute for Transtextual and Transcultural Studies (www.iett.eu). His main research interests include ideology and discourse in contemporary China, Chinese official and political language, technologies of power, and the discourses surrounding the idea of "civilisation". His PhD thesis, entitled "Towards a 'Harmonious' Consumer Society? The Discourse and Spectacle of Social Harmony in the Construction of a 'Civilized' China (1978-2008)", focused on Hu Jintao's concept of a "harmonious society" and its relation to the development of consumerism.

Articles du même auteur

La Chine en débat [Texte intégral]

Editorial [version française]

Paru dans Transtext(e)s Transcultures 跨文本跨文化 , 6 | 2011

Debating China [Texte intégral]

Editorial [English version]

Paru dans Transtext(e)s Transcultures 跨文本跨文化 , 6 | 2011

\section{Droits d'auteur}

() Tous droits réservés 